ARTICLE

Received 1 Aug 2014 | Accepted 21 Oct 2014 | Published 17 Dec 2014

DOI: $10.1038 /$ ncomms 6627

OPEN

\title{
Activated astrocytes enhance the dopaminergic differentiation of stem cells and promote brain repair through bFGF
}

Fan Yang ${ }^{1, \star}$, Yunhui Liu ${ }^{1, \star}$, Jie Tu ${ }^{1, \star}$, Jun Wan ${ }^{1}$, Jie Zhang ${ }^{1}$, Bifeng $\mathrm{Wu}^{1}$, Shanping Chen ${ }^{1}$, Jiawei Zhou ${ }^{2}$, Yangling $\mathrm{Mu}^{1, \dagger} \&$ Liping Wang ${ }^{1}$

Astrocytes provide neuroprotective effects against degeneration of dopaminergic (DA) neurons and play a fundamental role in DA differentiation of neural stem cells. Here we show that light illumination of astrocytes expressing engineered channelrhodopsin variant (ChETA) can remarkably enhance the release of basic fibroblast growth factor (bFGF) and significantly promote the DA differentiation of human embryonic stem cells (hESCs) in vitro. Light activation of transplanted astrocytes in the substantia nigra (SN) also upregulates bFGF levels in vivo and promotes the regenerative effects of co-transplanted stem cells. Importantly, upregulation of bFGF levels, by specific light activation of endogenous astrocytes in the SN, enhances the DA differentiation of transplanted stem cells and promotes brain repair in a mouse model of Parkinson's disease (PD). Our study indicates that astrocyte-derived bFGF is required for regulation of DA differentiation of the stem cells and may provide a strategy targeting astrocytes for treatment of PD.

\footnotetext{
${ }^{1}$ Shenzhen Key Lab of Neuropsychiatric Modulation, CAS Center for Excellence in Brain Science, Shenzhen Institutes of Advanced Technology, Chinese Academy of Sciences, Shenzhen 518055, China. ${ }^{2}$ State Key Laboratory of Neuroscience, CAS Center for Excellence in Brain Science, Institute of Neuroscience, Shanghai Institutes for Biological Sciences, Chinese Academy of Sciences, Shanghai 200031, China. ${ }^{\star}$ These authors contributed equally to this work. †Present address: Department of Physiology, Tongji Medical College, Huazhong University of Science and Technology, Wuhan 430030, China. Correspondence and requests for materials should be addressed to L.W. (email: Ip.wang@siat.ac.cn).
} 
D egeneration and dysfunction of dopaminergic (DA) neurons are involved in various neurodegenerative and mental disorders, such as Parkinson's disease (PD) and schizophrenia, for which effective therapeutic approaches are still being explored. Direct differentiation of embryonic stem cells (ESCs) intoDA neurons has been attained ${ }^{1,2}$ and may provide a source of cell transplantation therapy for PD treatment ${ }^{3}$. A major challenge in enhancing the therapeutic efficacy of ESCs is to promote their proper differentiation and long-term survival in the brain regions that are vulnerable to neurodegeneration in PD.

It has been shown that a number of important molecular pathways play key roles in DA neurogenesis, such as the sonic hedgehog (Shh) signalling ${ }^{4,5}$, Wnt/Catenin signalling ${ }^{6-8}$ and FGF signalling pathways ${ }^{9,10}$, which regulate the induction, differentiation and maturation of DA neurons. Basic fibroblast growth factor (bFGF), as a physiologically relevant neurotrophic factor, plays an essential role in embryonic development and neural lineage specification of ESCs ${ }^{11,12}$. It is one of the crucial elements specifying DA differentiation of ESCs that is widely used to induce the tyrosine hydroxylase $(\mathrm{TH})$-producing DA neurons ${ }^{13,14}$. Fibroblast growth factor receptors (FGFRs) have also been shown to cooperatively regulate the self-renewal and DA differentiation of neural progenitors in the developing midbrain $^{15}$. In $\mathrm{PD}$, a profound depletion of bFGF is found in $\mathrm{DA}$ neurons of the substantia nigra $(\mathrm{SN})^{16,17}$, whereas cotransplantation of bFGF-producing cells with DA neurons significantly enhances the graft survival and functional recovery 18,19 . Together, these findings support the notion that control of bFGF signalling may provide a useful means for optimizing ESC-based therapies for PD.

bFGF expression has been localized to both DA neurons and glial cells ${ }^{20}$. In the adult brain, bFGF is predominantly synthesized and secreted by astrocytes ${ }^{21}$. Nevertheless, it remains unclear whether astrocyte-derived bFGF is sufficient to induce DA differentiation of stem cells and thereby enhance brain repair in a PD model. Emerging studies have demonstrated that astrocytes play important roles in midbrain DA neuron development and modulation of adult neurogenic potential of neuroprogenitors ${ }^{22-24}$. Astrocytes could also secret trophic factors or signalling molecules to protect DA neurons from toxicity in a PD model ${ }^{6,25-30}$. The activation of astrocytes could suppress neuroinflammation and improve the resistance of DA neurons ${ }^{31,32}$. To enhance the DA differentiation of human EScs (hESCs), researchers have used midbrain astrocytes to substantially potentiate the DA differentiation of the hEScs, and the obtained DA implant yielded a significant restoration of motor function in the parkinsonian rats ${ }^{33}$, suggesting that the midbrain-specific astrocytes play an important role in promoting the differentiation of hESCs and functional recovery in the PD model. Here,we hypothesized that specific activation of the midbrain astrocyte population may increase the synthesis or release of bFGF, which may play a role in promoting the DA differentiation of transplanted stem cells and protecting the residual DA neurons in the PD model.

To test our hypothesis, we used an optogenetic tool to investigate the effects of specific activation of individual astrocyte populations on the DA differentiation of stem cells and the underlying mechanisms. We found that the light-activated astrocytes upregulated the synthesis of bFGF in a tissue-specific manner and significantly promoted the DA differentiation of the hESCs. Most importantly, to our knowledge, we are the first to demonstrate that specific activation of endogenous astrocytes in the SN through an optogenetic approach promoted the astrocytespecific bFGF release in situ, which substantially enhanced the DA differentiation of transplantedstem cells and promoted the regenerative effects in a mouse model of $\mathrm{PD}$.

\section{Results}

Activated astrocytes induced the differentiation of hESCs. To determine whether optogenetic manipulation of astrocytes could influence the neural differentiation of the human ESCs, we transfected the rat-brain-derived astrocytes (Fig. 1a) using a lentivirus carrying the CMV-ChETA-eYFP construct (Fig. 1b). At $48 \mathrm{~h}$ after the transfection, about $85.15 \%$ of the GFAP-positive astrocytes were successfully transfected to express ChETA-eYFP (Fig. 1c). We then used patch-clamp techniques to investigate the function of ChETA in the transfected astrocytes (Fig. 1d). Stimulating ChETA-expressing astrocytes with blue light (450-490 nm) for $500 \mathrm{~ms}$ induced depolarizing currents (Fig. 1e) with a peak amplitude at $142.9 \pm 37.7 \mathrm{pA}$ and a steady-state amplitude at $71.2 \pm 20.7 \mathrm{pA} \quad(n=6$, Supplementary Fig. 1b). We used $50 \mathrm{~ms}$ of light stimuli with a time interval of $50 \mathrm{~ms}$ to illuminate the astrocytes, and found that ChETA spike trains could be successfully induced by light stimulation (Fig. 1f), suggesting that the protocol we used was sufficient to induce the membrane depolarization of the ChETA-expressing astrocytes.

Then we constructed a blue-light-emitting diode (LED) to illuminate the transfected astrocytes for $1 \mathrm{~h}(10 \mathrm{~Hz}$ and $50-\mathrm{ms}$ stimulus intervals), and the conditioned medium (CM) was collected $24 \mathrm{~h}$ after the light stimulation (Fig. 1g). To investigate the effects of CM on neural differentiation of human ESCs, we used human ESC line 9, which expressed pluripotency markers including SSEA3, SSEA4, Tra60 and Tra81 (Fig. 1h). On stimulation with CM from optogenetic-activated astrocytes and other differentiation components, the hESCs gradually differentiated into cobblestone-like cells and then spindled neural progenitors (Fig. 1i,j). Nestin and Tuj1 immunofluorescence staining was performed to evaluate the differentiation efficiency. There were significant increases in the numbers of nestin-positive and Tuj1-positive cells in the ChETA + light group compared with Control and eYFP + light groups (Fig. 1i,j). We also studied the effect of CM on the proliferative potential of hESCs:the 5-ethynyl-2-deoxyuridine (EdU) incorporation assay showed that the percentage of EdU-positive cells in the ChETA + light group increased significantly compared with Control group (Supplementary Fig. 2d). A co-culture system was set up to study the effect of optogenetic-activated astrocytes on the stem cell differentiation process:the astrocytes expressing ChR2 or eYFP were co-cultured with stem cells and stimulated with blue light (Supplementary Fig. 2a). We observed that the percentage of nestin-positive and Tuj1-positive cells in ChETA + light group increased significantly compared with Control and eYFP + light groups (Supplementary Fig. 2b,c), further supporting the suggestion that optogenetic-activated astrocytes could enhance the neural differentiation of human ESCs.

We further investigated the effects of CM from optogeneticactivated astrocytes on the DA differentiation of stem cells: the EdU incorporation assay showed that the percentage of EdUpositive cells was increased significantly in the ChETA + light group (Supplementary Fig. 2e), and the typical morphology of DA neurons was observed at the late stage of differentiation (Fig. 2a). The dual staining of Tuj1 and TH at different stages of the differentiation process showed increased positive signals of Tuj1 and TH with time, supporting the progressive differentiation of DA neurons (Fig. 2a). Quantitative real-time PCR analysis showed upregulated gene expression of Tuj1, Nurr,TH and DAT in the ChETA + light group compared with those in the Control and eYFP + light groups (Fig. 2b), confirming the DA differentiation of stem cells. Strong expression of TH and dopamine transporter (DAT), markers for DA neurons was observed in the differentiated hES cells (Fig. 2c,d). The percent of TH-positive and DAT-positive cells in the ChETA + light group was 
$34.54 \pm 1.77$ and $40.97 \pm 1.77 \%$, respectively (Fig. $2 c, d$ ), which were significantly higher than those in the Control and eYFP + light groups, suggesting the optogenetic-activated astrocytes could significantly enhance the DA differentiation of ESCs. To test the electrophysiological properties of differentiated DA cells, we performed whole-cell patch-clamp recordings (Fig. 2e) and observed that the recorded cells exhibited action potentials on injection of depolarizing currents (Fig. 2e). We also recorded the hyperpolarization-activated inward current $\left(I_{\mathrm{h}}\right)$ (Fig. 2e), which is a common feature of DA neurons ${ }^{34}$. To further evaluate the function of the obtained DA neurons, the differentiated DA neurons were transplanted into the $\mathrm{SN}$ region of the MPTP

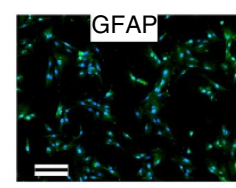

d

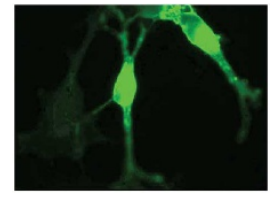

b

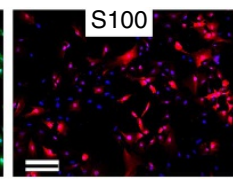

C

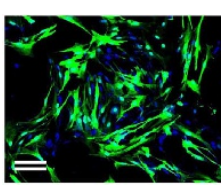

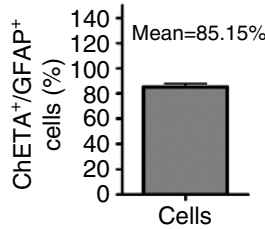

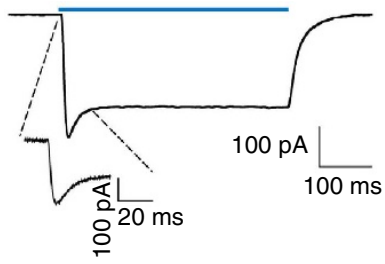

f

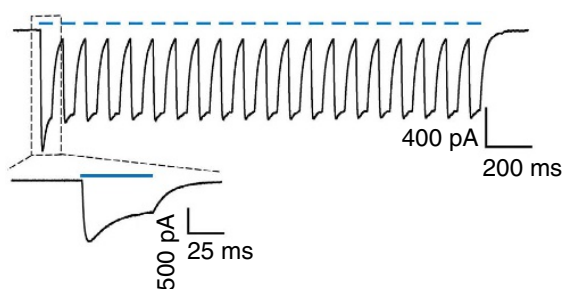

g

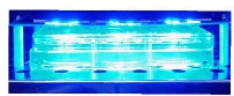

Light-emitting diode

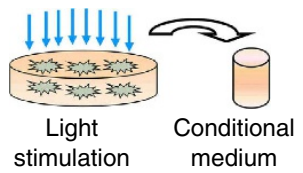

stimulation medium h

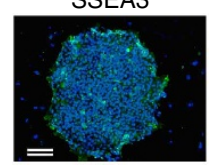

SSEA4

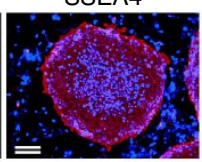

Tra81

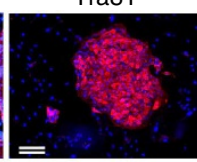

Tra60

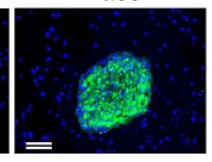

i
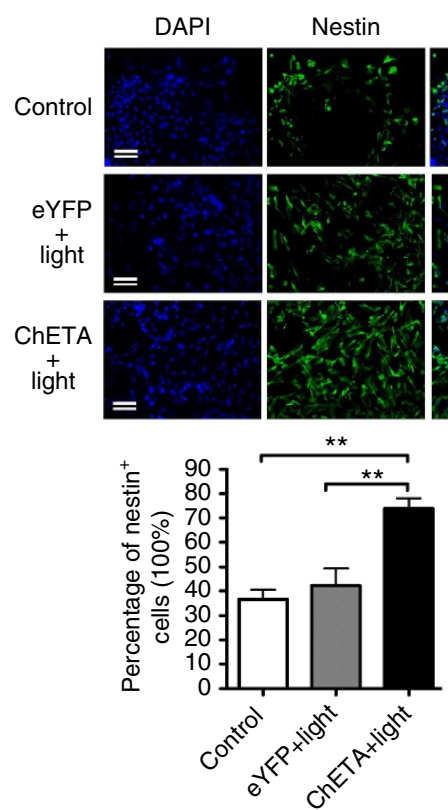

Merge

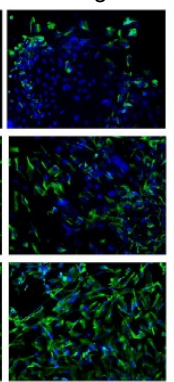

j

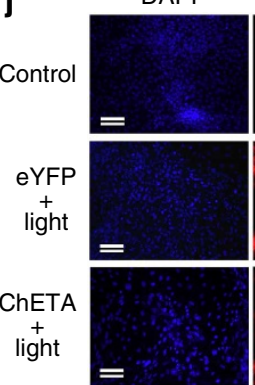

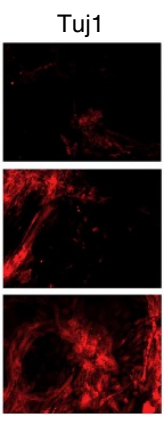

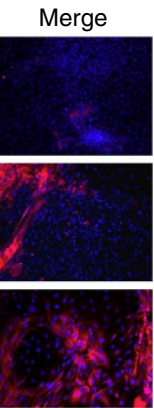

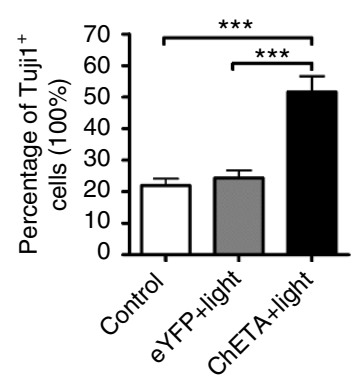

Figure 1 | Light stimulation of astrocytes induced the neural differentiation of human embryonic stem cells. (a) Immunofluorescence of glial fibrillary acidic protein (GFAP) and S100 on cultured rat-brain-derived astrocytes. Bar, $50 \mu \mathrm{m}$. (b) Schematic drawing of the CMV-ChETA-eYFP lentiviral vector construct. (c) Positive ChETA expression (green) was observed in the astrocytes transfected with CMV-ChETA-eYFP lentivirus (left panel). The percentage of ChETA-expressing astrocytes over the total GFAP-positive cells (right panel); mean \pm s.e.m. Bar, $25 \mu \mathrm{m}$. (d) Whole-cell patchclamp was used to record light-evoked photo currents from ChETA-expressing astrocytes. (e) Light stimulation $(500 \mathrm{~ms})$ induced depolarization and a recovery current. (f) ChETA channel current spike trains induced by light pulses $(50 \mathrm{~ms}, 10 \mathrm{~Hz}$ ). (g) The constructed blue-light- emitting diode was used to illuminate the cultured astrocytes and the conditioned medium was collected after the light stimulation. (h) Immunofluorescence of SSEA3, SSEA4, Tra81 and Tra60 on the human embryonic stem cells (hESCs); Bars, $50 \mu \mathrm{m}$. (i) Immunostaining of Nestin in Control, eYFP + light and ChETA + light groups. The percentage of Nestin-positive cells in the ChETA + light group increased compared with the Control and eYFP + light groups. Bars, $50 \mu \mathrm{m}$, (mean \pm s.e.m., $n=10)$. (j) Immunostaining of Tuj1 in the Control, eYFP + light and ChETA + light groups. The percentage of Tuj1-positive cells in the ChETA + light group increased compared with the Control and eYFP + light groups. Bar, $50 \mu \mathrm{m}$, (mean \pm s.e.m., $n=10$ ). All analyses based on Student's $t$-test, ${ }^{\star \star} P<0.01$; ${ }^{\star * \star} P<0.001$. The experiments were repeated three times with similar results. 
a
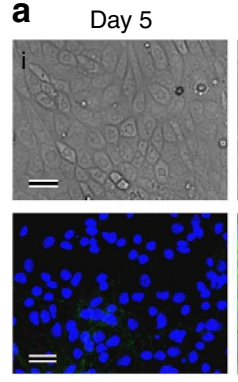

Hoechst Tuj1 TH
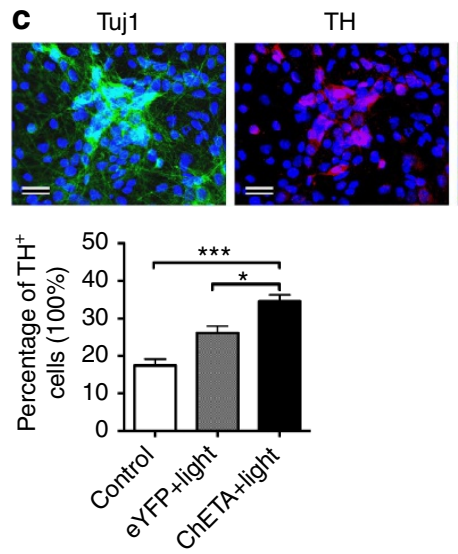

e
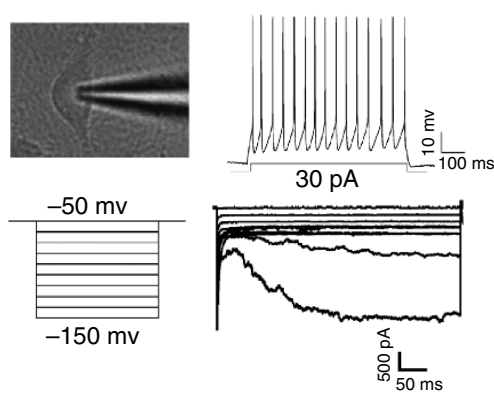

Day 15
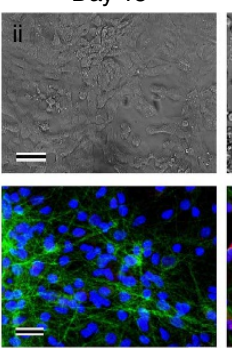

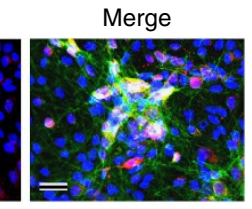

f

Differentiated dopaminergic neurons
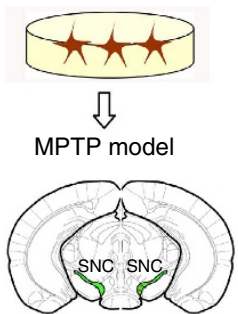

b
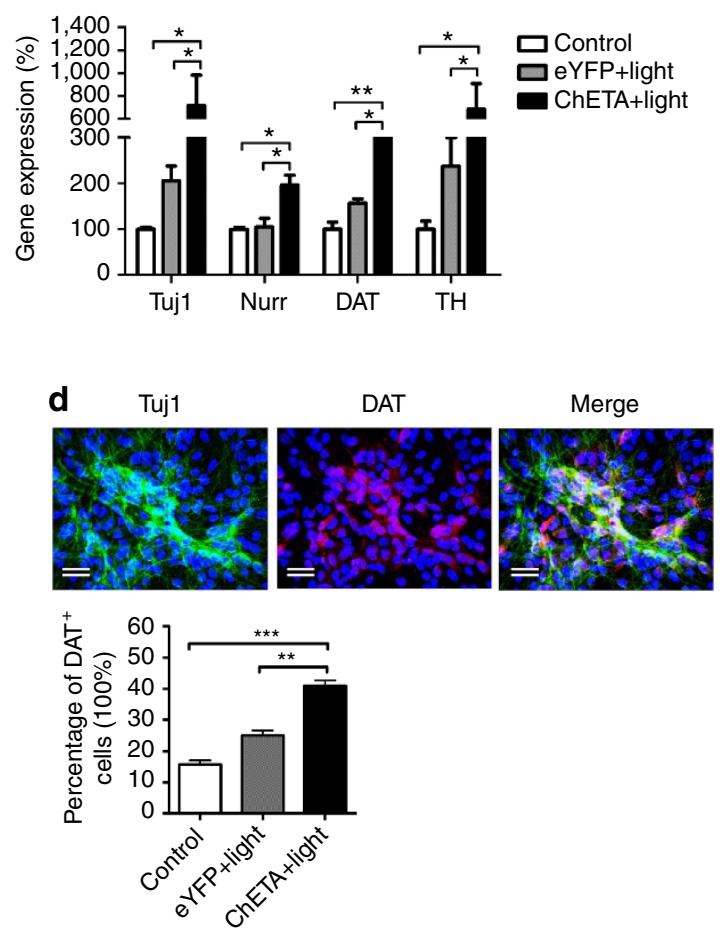

g
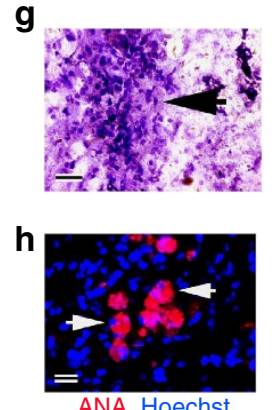

ANA Hoechst i Non-transplanted

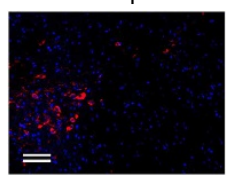

Transplanted

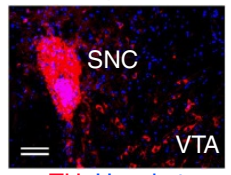

TH Hoechst

Figure 2 | ChETA expression and light stimulation of astrocytes induced the dopaminergic differentiation of human embryonic stem cells.

(a) hESCs differentiated gradually from cobblestone-like cells (I) into spindled neural progenitors (II) and then typical dopaminergic neurons (III) under the stimulation of conditioned medium from astrocytes (top panel). The dual staining of Tuj1 and TH revealed the progressively differentiated dopaminergic neurons (bottom panel). Bars, $20 \mu \mathrm{m}$. (b) qRT-PCR analysis showed that gene expression of Tuj1, Nurr, Dat and TH increased significantly in the ChETA + light group compared with the Control and eYFP + light groups (mean \pm s.e.m., $n=3$ ). (c) Immunostaining of tyrosine hydroxylase (TH) in differentiated dopaminergic neurons. The percentage of TH-positive cells in the ChETA + light group increased compared with the Control and eYFP + light groups (mean \pm s.e.m., $n=8$ ). Bars, $20 \mu \mathrm{m}$. (d) Immunostaining of dopamine transporter (DAT) in differentiated dopaminergic neurons. The percentage of DAT-positive cells in the ChETA + light group increased compared with the Control and eYFP + light groups (mean \pm s.e.m., $n=8$ ). Bars, 20 $\mu \mathrm{m}$.

(e) Whole-cell patchclamp was used to record action potentials from differentiated dopaminergic neurons. The injection of depolarizing currents ( $30 \mathrm{pA}$ ) to dopaminergic neurons led to the firing of action potentials under current-clamp conditions (top panel). The hyperpolarization-activated inward current ( $I_{\mathrm{h}}$ ) was recorded from the dopaminergic neurons during the hyperpolarization steps (bottom panel). (f) Schematic flowchart of the transplantation of the differentiated dopaminergic neurons. (g) Representative histological analysis (HE staining) of substantia nigra (SN) at 4 weeks after the transplantation. Bars, $50 \mu \mathrm{m}$. (h) Anti-nuclear antibody (ANA) staining reveals the origin of the transplanted dopaminergic neurons. Bars, $20 \mu \mathrm{m}$. (i) Immunostaining of TH of the transplanted and non-transplanted side of substantia nigra. Strong signal should be detected on the transplant $(A P=-3.28, M L=-1.5, D V=4.2)$. Bars, $50 \mu \mathrm{m}$. All analyses based on Student's $t$-test. ${ }^{\star} P<0.05 ;{ }^{\star \star} P<0.01 ;{ }^{\star \star \star} P<0.001$. The experiments were repeated three times with similar results.

mouse model of PD (Fig. 2f). Four weeks after transplantation, H\&E staining showed that cell grafts were positioned within the target brain region (Fig. $2 \mathrm{~g}$ ), and their human origin was confirmed by anti-nuclear Antibody (ANA) staining (Fig. 2h), $\mathrm{TH}$ immunofluorescent staining revealed that there were few $\mathrm{TH}$ signals in the non-transplanted $\mathrm{SN}$ region, while strong $\mathrm{TH}$ signals were observed in the transplanted $\mathrm{SN}$ region (Fig. 2i). Dopamine level in the treatment group increased significantly compared with the MPTP group (Supplementary Fig. 3a), and motor function assays showed that the transplanted mice had an improved forelimb strength (Supplementary Fig. 3b), a shorter time to orient down and descend in the Pole Test (Supplementary Fig. 3c,d), and a longer distance of locomotion and higher velocity in the OpenField test (Supplementary Fig. 3e,f) compared with the MPTP control group. TH staining in the striatum showed that TH-positive signals increased in the transplanted side compared with the non-transplanted side of the striatum (Supplementary Fig. 3g). Taken together, these data consistently demonstrated 
that the optogenetic-activated astrocytes could enhance the DA differentiation of human ESCs into functional DA neurons, which promoted brain repair in an MPTP model.

Light stimulation promoted the synthesis of astrocytic bFGF. SHH, FGF8 and bFGF have beencommonly used to induce the DAprogenitors from human $\mathrm{ESCs}^{33}$. We investigated whether ChETA expression and light stimulation could indeed enhance the release of SHH, FGF8 and bFGF from astrocytes: light stimulation did not significantly alter the levels of SHH and FGF8 in $\mathrm{CM}$ from astrocytes. However the bFGF level in the ChETA + light group was significantly elevated compared with Control and eYFP + light groups (Fig. 3a). The levels of bFGF in $\mathrm{CM}$ increased significantly from 6 to $12 \mathrm{~h}$, followed by a decline at $24 \mathrm{~h}$ after the light stimulation (Supplementary Fig. 1c). To exclude the possibility that bFGF elevation is due to cell membrane leakage, we quantified lactic dehydrogenase (LDH) release, and found that there was no significant difference among the three groups (Supplementary Fig. 1d). Next we investigated the biosynthesis of bFGF in astrocytes upon light stimulation. Western blotting showed that the expression level of bFGF was upregulated in the ChETA + light group, and the bFGF expression level in the ChETA + light group was significantly higher than that of Control and eYFP + light groups (Fig. 3b). To verify that light stimulation had indeed upregulated the synthesis of bFGF in ChETA-transfected astrocytes, we performed siRNA knockdown experiments in ChETA-expressing astrocytes. We found that the bFGF expression level after the light stimulation was significantly decreased when the astrocytes were pre-treated with bFGF-siRNAs (siRNA1 and siRNA2), compared with those treated with control siRNAs (Fig. 3c). The level of bFGFassociated signalling molecules, including protein kinase A (PKA) and cyclic AMP (cAMP), was also significantly increased in the ChETA + light group compared with those of the Control and eYFP + light groups (Fig. 3d,e), suggesting that light stimulation activated the PKA/cAMP signalling pathway to promote bFGF synthesis in the transfected astrocytes.

To confirm that bFGF derived from astrocytes ${ }^{35}$ is necessary for the neuronal differentiation of human ESCs, the CM was incubated with an anti-bFGF antibody to neutralize the bFGF (Fig. 3f-h). We found that the bFGF antibody effectively attenuated the neural and DA differentiation capacity of astrocyte-derived CM, indicated by the significantly decreased number of nestin-positive, Tuj1-positive and TH-positive cells in the $\mathrm{CM}$ from the $\mathrm{CM}+\mathrm{Ab}$ group (Fig. $3 \mathrm{f}-\mathrm{h}$ ). Importantly, the decreased neural/DA differentiation capacity of CM could be significantly rescued when exogenous bFGF was added into the previously neutralized CM (Fig. 3f-h). Taken together, these data suggest that bFGF released into astrocytic medium is essential for the neural and DA differentiation of the human ESCs.

Activated astrocytes enhanced the repair of stem cells. To investigate whether light stimulation of ChETA-transfected astrocytes could upregulate bFGF level in vivo to enhance the differentiation of stem cells, ChETA-transfected astrocytes along with neural progenitors derived from human ESCs were prepared, and co-transplanted into the $\mathrm{SN}$ of the MPTP-mouse model of PD (Fig. 4a,b, Supplementary Fig. 4a). One week after the transplantation, the ANA immunofluorescence and eYFP fluorescence confirmed the viability of the co-transplanted cells (Fig. 4c). Then we used a blue-light-emitting optical fibre to illuminate the $\mathrm{SN}$ region for $30 \mathrm{~min}$ with an average light illumination output power of $10 \mathrm{~mW} \mathrm{~mm}^{-2}$ (Fig. 4d). ELISA analysis showed that 1 week after the light stimulation, the bFGF level in the ChETA + light group increased significantly compared with the Control and eYFP + light groups (Fig. 4e). To verify that the elevated bFGF was mainly derived from cotransplanted astrocytes, we also co-transplanted astrocytes that had been treated with siRNA targeting bFGF; the bFGF level in the ChETA + light + siRNA group decreased significantly compared with the ChETA + light group (Fig. 4e). Then we investigated the change of endogenous DA neurons at each phase after the co-transplantation and light stimulation (Supplementary Fig. 4a). We found that the number of TH-positive cells decreased significantly in MPTP Control, ChETA + light, ChETA + light + SiRNA groups at 7 days after MPTP; however there were more TH-positive cells in the ChETA + light group compared with the other groups after the light stimulation (Supplementary Fig. 4b,c). Light stimulation significantly increased striatal dopamine level (Supplementary Fig. 5a) and improved motor function in the ChETA + light group (Supplementary Fig. 5b-f), including increased forelimb grip strength (Supplementary Fig. 5b), reduced time to orient down and descend (Supplementary Fig. 5c,d), and increased distance of locomotion and average velocity (Supplementary Fig. 5e,f). TH staining in the striatum showed that $\mathrm{TH}$ signals increased in the transplanted side of striatum compared with the nontransplanted side (Supplementary Fig. 5g), supporting that light stimulation of co-transplanted cells in sustantianigra promoted functional brain repair in the MPTP model

To evaluate whether elevated astrocytic bFGF influenced the DA differentiation of transplanted stem cells, we performed dual staining of ANA and TH. ANA/TH-positive signals were coexpressed in the transplanted stem cells (Fig. 4f). Quantitative analysis showed that the fold changes of $\mathrm{TH}^{+} / \mathrm{ANA}^{+}$cells in eYFP + light, ChETA + light and ChETA + light + siRNA groups were $1.00 \pm 0.14,1.88 \pm 0.28$ and $0.91 \pm 0.13$ respectively, and the number of $\mathrm{TH}^{+} / \mathrm{ANA}^{+}$cells was significantly increased in the ChETA + light group and decreased in the ChETA + light + siRNA group $(P<0.01)$ (Fig. $4 \mathrm{~g})$.

We also evaluated the effects of intrastriatal co-transplantation of ChETA-transfected astrocytes and ES-derived stem cells in both MPTP and 6-OHDA models after the light stimulation. In the MPTP model, DA differentiation of transplanted stem cells was observed in the striatum (Supplementary Fig. 6a).Dopamine levels in the ChETA + light group were increased significantly compared with the MPTP group (Supplementary Fig. 6b). Motor function assays showed that the co-transplanted mice had an increased forelimb strength (Supplementary Fig. 6c), a shorter time to orient down and descend (Supplementary Fig. 6d,e), a longer distance of locomotion and higher velocity (Supplementary Fig. 6f,g) compared with the MPTP control and eYFP + light groups. In the 6-OHDA model, TH-positive cells were observed among the transplanted stem cells (Supplementary Fig. 7b). The bFGF levels in the ChETA + light group increased significantly (Supplementary Fig. 7c), which was accompanied by elevation of dopamine levels in the ChETA+ light group compared with the Control and eYFP + light groups (Supplementary Fig. 7d). Taken together, our data consistently demonstrate that light stimulation specifically upregulated the level of bFGF derived from transplanted astrocytes, enhanced the DA differentiation of stem cells and promoted brain repair in a mouse PD model.

Activation of endogenous astrocytes enhanced bFGF release. Endogenous astrocytes play important roles in regulating the local microenvironmentand influencing the fate of the stem cells ${ }^{36}$. To stimulate the endogenous astrocytes in a cell-typespecific manner, mouse brains were transfected with a construct expressing ChETA-eYFP under the control of GFAP promoter to 

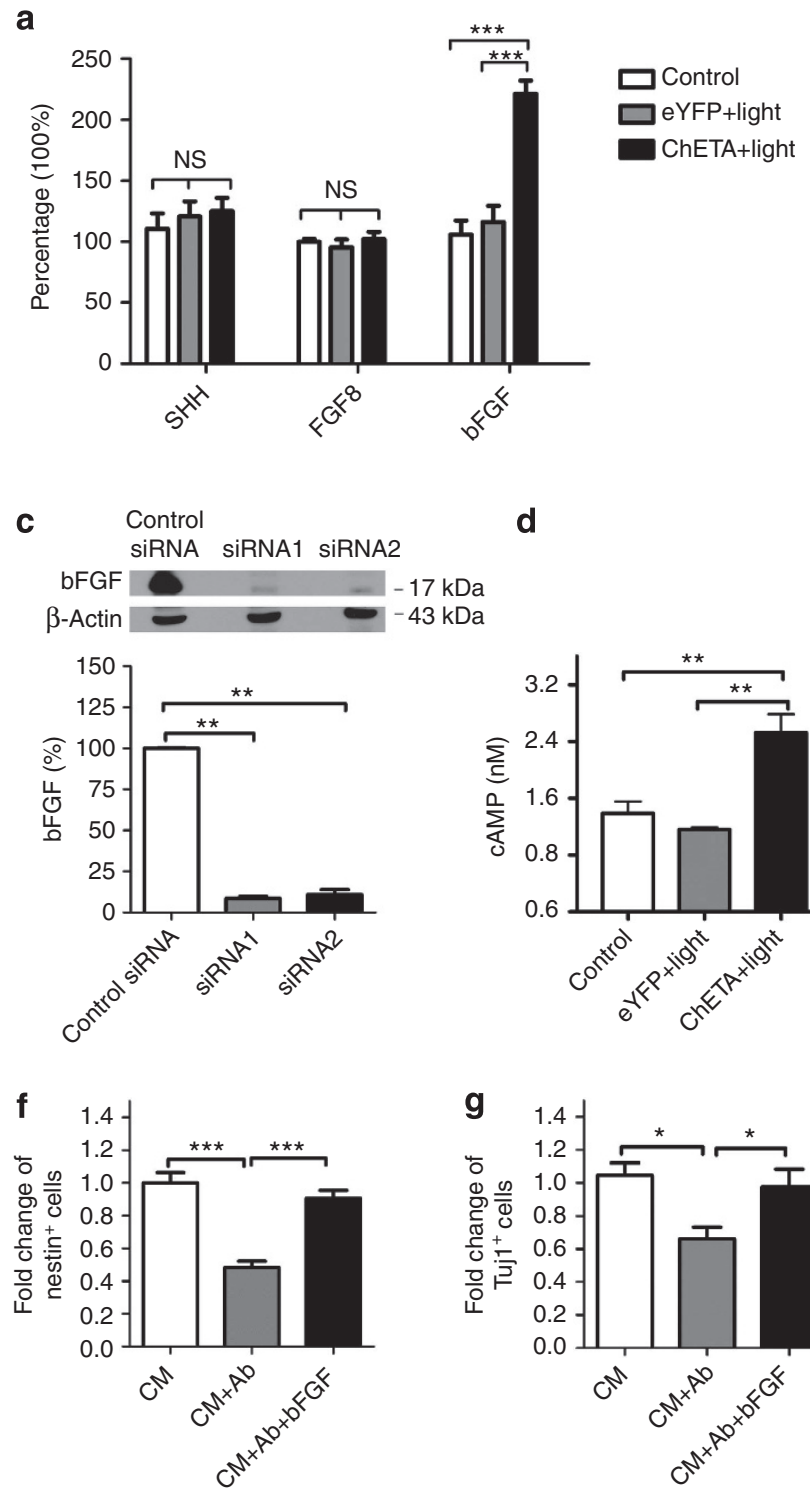

d $17 \mathrm{kDa}$
$-43 \mathrm{kDa}$
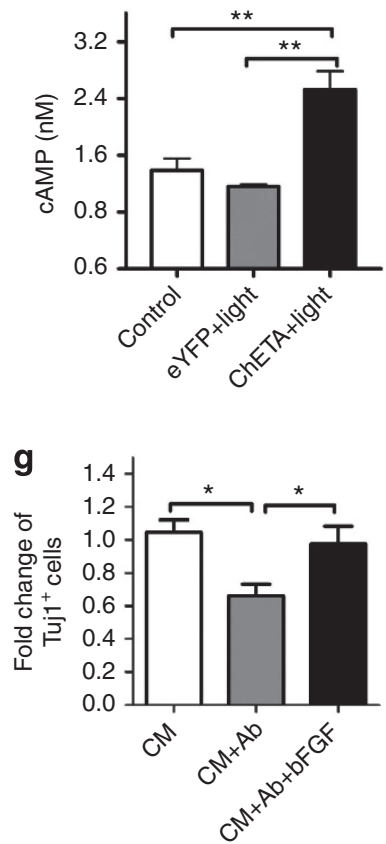

b Control eYFP+light ChETA+light
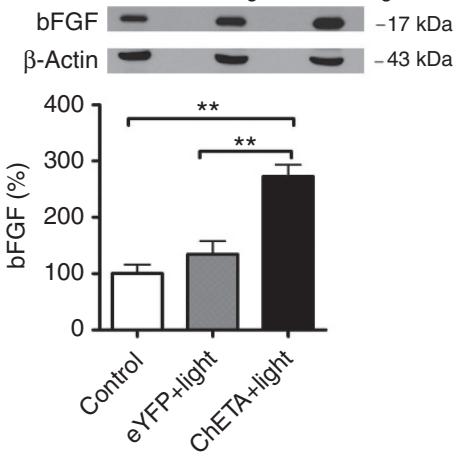

e
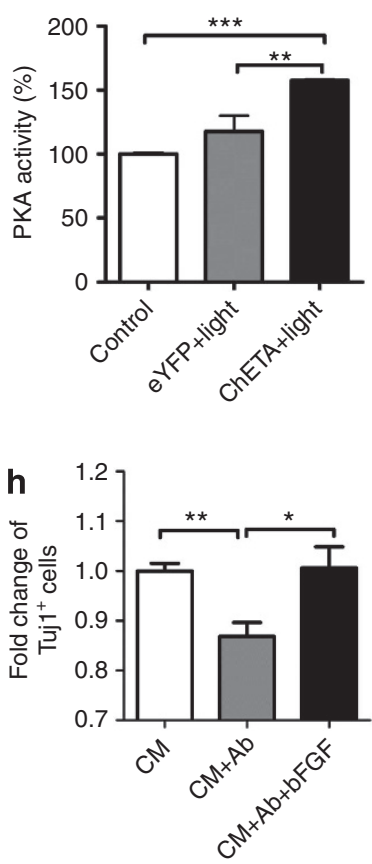

Figure 3 | Light stimulation of astrocytes increased the synthesis and release of bFGF. (a) Quantification of Sonic hedgehog (SHH), FGF8 and bFGF in the conditioned medium from astrocytes in the Control, eYFP + light and ChETA + light groups (mean \pm s.e.m., $n=6$ ). (b) Western blot of $\beta$-actin and bFGF protein expression in the Control, eYFP + light and ChETA + light groups (upper panel); quantification of bFGF protein expression in the Control, eYFP + light and ChETA + light groups (lowerpanel) (mean \pm s.e.m., $n=3$ ). (c) Western blot of bFGF and $\beta$-actin protein expression in the ChETA + light group treated with two separate bFGF-siRNAs (top panel); quantification of bFGF protein expression in the Control and siRNA-treated groups (bottom panel) (mean \pm s.e.m., $n=3$ ). (d) Quantification of cAMP in the conditioned medium from astrocytes in the Control, eYFP + light and ChETA + light groups (mean \pm s.e.m., $n=5$ ). (e) Quantification of Protein Kinase A (PKA) in the conditioned medium from astrocytes in the Control, eYFP + light and ChETA + light groups (mean \pm s.e.m., $n=5$ ). (f) The fold change of Nestin-positive cells in $C M, C M+A b$ and $C M+A b+b F G F$ groups (mean \pm s.e.m., $n=8$ ). Ab, anti-bFGF antibody; CM, conditioned medium. (g) The fold change of Tuj1-positive cells in CM, CM $+\mathrm{Ab}$ and $\mathrm{CM}+\mathrm{Ab}+\mathrm{bFGF}$ groups (mean \pm s.e.m., $n=8$ ). (h) The fold change of TH-positive cells in CM, CM + Ab and CM $+A b+b F G F$ groups (mean \pm s.e.m., $n=8$ ). All analyses based on Student's $t$-test. ${ }^{\star} P<0.05 ;{ }^{\star \star} P<0.01 ;{ }^{\star \star \star} P<0.001$. The experiments were repeated three times with similar results.

specifically target expression to astrocytes (Fig. 5a). Before the in vivo study, expression of ChETA was validated in primary cultured astrocytes. Expression of ChETA was seen 1 week after the transfection (Fig. 5b), and ChETA spike trains were successfully induced by light stimulation (Fig. 5c), indicating that there was functional expression of ChETA in astrocytes. Blue light stimulation could also significantly increase the released bFGFfrom ChETA-transfected astrocytes (Fig. 5d). Then we investigated the specific expression pattern of ChETA following injection of the GFAP-ChETA-eYFP lentiviral construct into the SN region of the MPTP mouse model. TH staining was performed at 1 week after the injection, and we found that there were few TH-positive signals (red) in the control group: the ChETA signals (green) were mainly concentrated in the SNc region interspersed with residue TH-positive neurons (red) (Fig. 5e). High magnification showed that most of the THpositive immunosignals were not localized in the ChETA-positive cells (Fig. 5h), indicating that the residual DA neurons in the SN region were not transfected by the GFAP-ChETA-eYFP construct. Then we investigated the specific expression of ChETA in endogenous astrocytes through GFAP staining. Strong GFAPpositive signals (red) were observed in the $\mathrm{SNc}$ region of the control group (Fig. 5f), and most of the ChETA signals (green)were concentrated in the $\mathrm{SNc}$ region where they were 
a
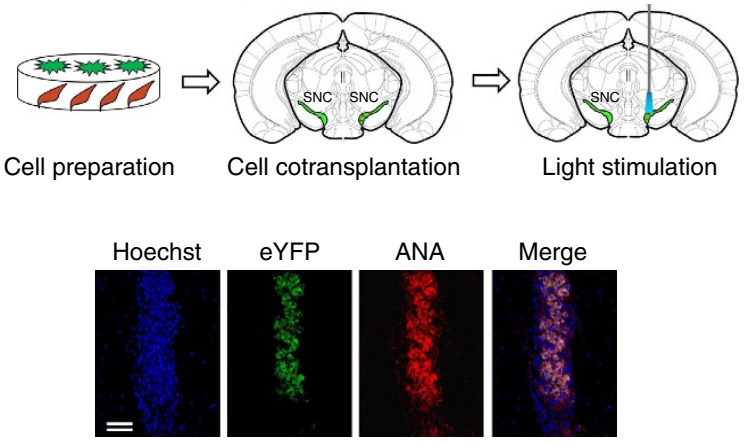

f

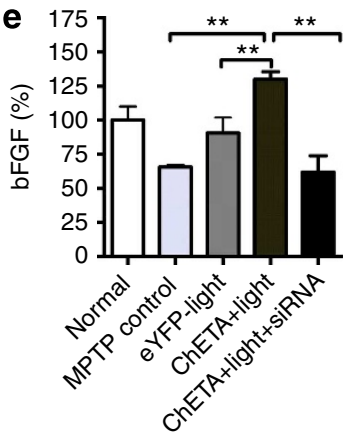

b

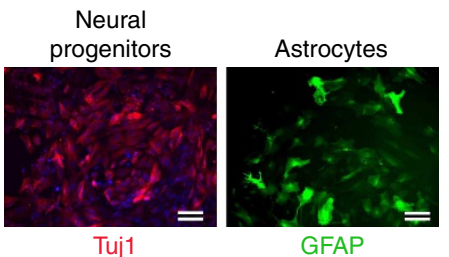

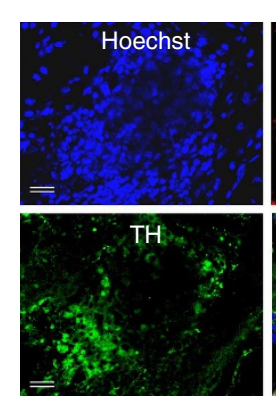

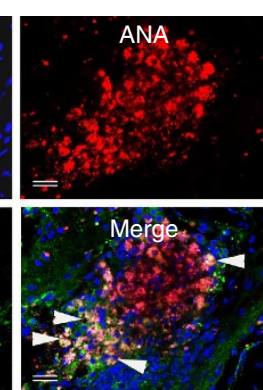

d

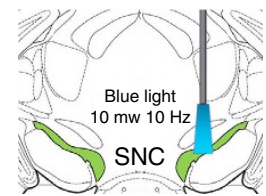

Figure 4 | Light stimulation of the transplanted astrocytes enhanced the regenerative capacity of the co-transplanted stem cells. (a) Schematic flowchart of the co-transplantation experiment. (b) Prepared ChETA-transfected astrocytes (green) and neural progenitors that express Tuj1 (red). Bars, $20 \mu \mathrm{m}$. (c) Co-localization of the viable transplanted cells using Anti-nuclear Antibody (ANA, red) staining and eYFP fluorescence (green) Bars, $150 \mu \mathrm{m}$. (d) Schematic drawing of illumination of cells in substantia nigra (SN) region using blue-light-emitting optical fibre. (e) Quantification of bFGF level from the SN tissue in the Normal, MPTP Control, eYFP + light, ChETA + light, ChETA + light + siRNAgroups. (mean \pm s.e.m., $n=6$ ). (f) Immunostaining of TH and ANA revealed the double-positive cells (arrows) among the transplanted stem cells. Bars, $40 \mu \mathrm{m}$. (g) The fold change of $\mathrm{TH}^{+} / \mathrm{ANA}^{+}$cells in co-transplanted stem cells from the MPTP Control, eYFP + light, ChETA + light and ChETA + light + siRNA groups (mean \pm s.e.m., $n=8$ ). All analyses based on Student's $t$-test. ${ }^{\star \star} P<0.01 ;{ }^{\star \star \star} P<0.001$. The experiments were repeated three times with similar results.

co-localized with GFAP fluorescent signals (Fig. 5f,i). Quantification showed that about 95\% of the ChETA-positive astrocytes expressed GFAP (Fig. 5g), suggesting that specific ChETA expression in endogenous astrocytes in the SN could be achieved through in vivo transfection.

Next we investigated whether light stimulation of endogenous ChETA-transfected astrocytes could promote functional repair in a PD model. First we found that the bFGF level in the ChETA+ light group increased significantly compared with the Control and eYFP + light groups (Fig. 5j). To confirm the elevated bFGF expression in the SN, bFGFimmunofluoresence was also performed after the light stimulation. bFGF immunosignals (red) were mainly concentrated in the region containing ChETA-transfected astrocytes (green) (Fig. 5k); whereas few bFGF signals were observed outside the region containing ChETA-expressing cells (Fig. 5k). High magnification showed that the strong positive bFGF signals were detected within the ChETA-positive astrocytes (Fig. 51). The level of cAMP was also significantly increased in the ChETA + light group compared with that of the Control and eYFP + light groups (Fig. $5 \mathrm{~m}$ ). After the light stimulation, the number of endogenous $\mathrm{TH}$ positive cells in the ChETA + light group increased significantly compared with those of other groups (Supplementary Fig. 8a,b). Dopamine levels in the ChETA + light group also increased significantly compared with the MPTP group (Supplementary Fig. 8c). Motor function assays showed that the treated mice exhibited increased forelimb strength (Supplementary Fig. 8d), a shorter time to orient down and descend in the pole test (Supplementary Fig. 8e,f), and a longer distance of locomotion and higher velocity in the open field test (Supplementary Fig. 8g,h) compared with MPTP control and eYFP + light groups. Taken together, these data demonstrate that the light stimulation specifically activated the endogenous astrocytes in vivo and significantly promoted functional brain repair inthe $\mathrm{PD}$ model.

Astrocytes enhanced the DA differentiation and brain repair. To investigate the influence of optogenetic-activated astrocytes on the activities of the transplanted stem cells, we expressed ChETA in endogenous astrocytes, transplanted the ES cell-derived neural progenitors and stimulated the $\mathrm{SN}$ region using blue light (Fig. 6a,b). Three weeks after the sequential light illumination, the transplanted stem cells (red) were identified with ANA staining, and we found that there were more ANA-positive cells in the ChETA + light group compared with the Control and eYFP + light groups. However, the positive signals were decreased in the FGFR inhibitor SU5402-treated group (Fig. 6c). Quantification showed that the number of ANA-positive cells was significantly increased in the ChETA + light group and decreased in the FGFR inhibitor group (Fig. 6d). To further investigate the differentiation of the transplanted stem cells, $\mathrm{TH}$ immunofluorescence and ANA immunofluorescence were recorded simultaneously. We found positive expression of TH among the ANA-positive cells (Fig. 6e). Quantification showed that the numbers of $\mathrm{TH}^{+} / \mathrm{ANA}^{+}$cells were significantly increased in the ChETA + light group compared with the Control group and decreased in the FGFR inhibitor group (Fig. 6f), suggesting that light stimulation effectively 
a
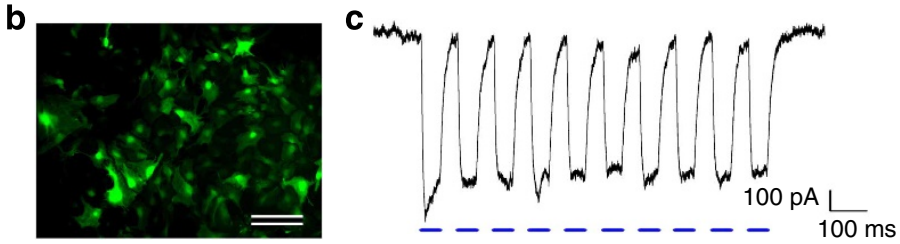
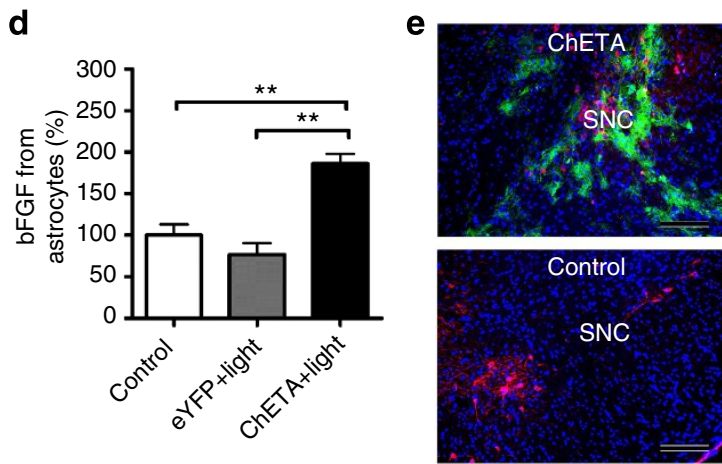

TH ChETA

h
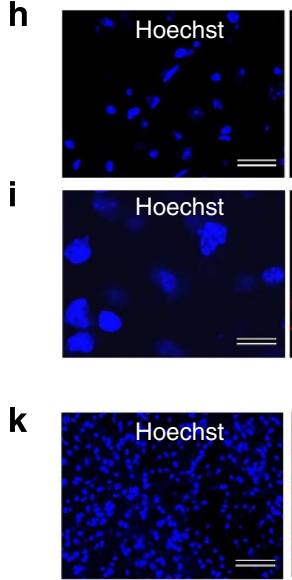

I

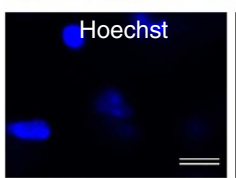

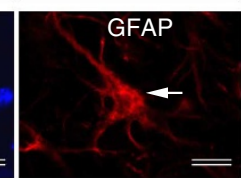
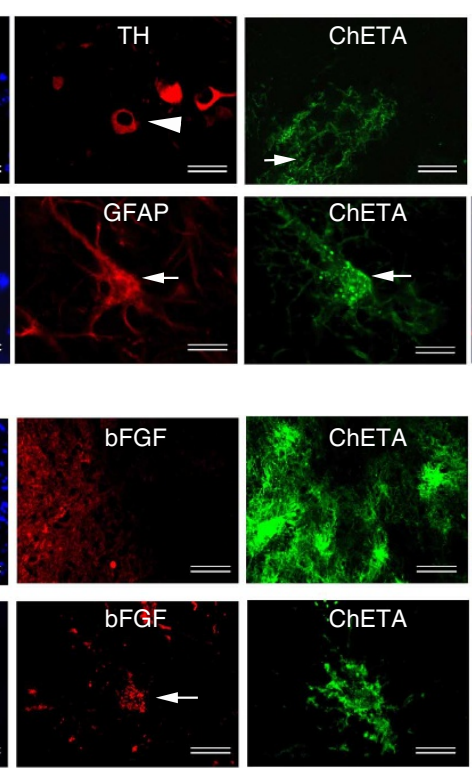

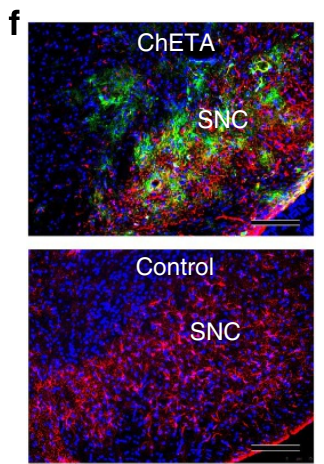

g

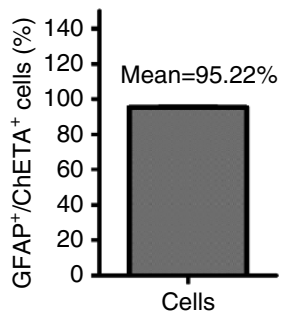

GFAP ChETA
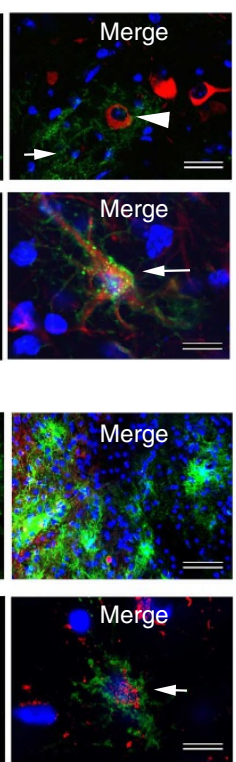

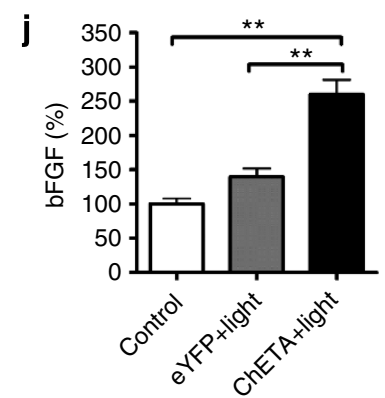

m

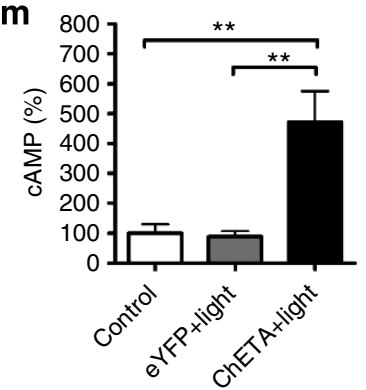

Figure 5 | Expression of ChETA in endogenous astrocytes and light stimulation enhanced the bFGF release in vivo. (a) Schematic drawing of the GFAP-ChETA-eYFP lentivirus vector constructs. (b) Positive ChETA expression (green) was observed in the astrocytes transfected with GFAP-ChETA-eYFP lentivirus. (c) ChETA channel current spike trains induced by light pulses $(50 \mathrm{~ms}, 10 \mathrm{~Hz})$. (d) Quantification of bFGF in the conditioned medium from astrocytes in the Control, eYFP + light and ChETA + light groups (mean \pm s.e.m., $n=6)$. (e) Immunostaining of tyrosine hydroxylase $(\mathrm{TH}$, red) and in vivo expression of ChETA (green) in ChETA group and Control group after injecting the GFAP-ChETA-eYFP lentivirus into substantia nigra (SN) region. Bars, $80 \mu \mathrm{m}$. (f) Immunostaining of GFAP (red) and in vivo expression of ChETA (green) in ChETA group and Control group. Bars, $80 \mu \mathrm{m}$. (g) The percentage of GFAP-positive cells in the total number of ChETA-positive cells, $n=10$. (h) High magnification of immunostaining of TH (red, arrowhead) and ChETA (green, arrow) expression in SN. Bars, $20 \mu \mathrm{m}$. (i) High magnification of immunostaining of GFAP (red) and ChETA (green) expression in transfected astrocytes. Bars, $10 \mu \mathrm{m}$. (j) Quantification of bFGF from SN tissue in the Control, eYFP + light and ChETA + light groups (mean \pm s.e.m., $n=6$ ).

(k) Immunostaining of bFGF after the light stimulation. The signals (red) were concentrated in the left region occupied by positive ChETA-expressing cells (green). Bars, $50 \mu \mathrm{m}$. (I) High magnification of immunostaining of bFGF (red) and expression of ChETA (green) in transfected astrocytes. Bars, $10 \mu \mathrm{m}$. (m) Quantification of cAMP in the SN region in the Control, eYFP + light and ChETA + light groups (mean \pm s.e.m., $n=6$ ). All analyses based on Student's $t$-test. ${ }^{\star \star} P<0.01$. The experiments were repeated three times with similar results.

promoted the bFGF dependent-DA differentiation of the transplanted stem cells.

Then we evaluated the activity of endogenous DA neurons after the light stimulation. We found that there were more $\mathrm{TH}$ positive (but ANA-negative) cells in the ChETA + light group compared with the Control and eYFP + light groups; however, the fluorescence intensity decreased in SU5402-treated animals
(Fig. 6g). The number of TH-positive cells was significantly increased in the ChETA + light group and decreased in SU5402treated animals (Fig. 6h), suggesting that light stimulation and stem cell transplantation prevented the decrease of the endogenous DA neurons in the PD model.

The 6-OHDA mouse model of PD (Supplementary Fig. 7a) was used to evaluate functional recovery after optogenetic activation 
a

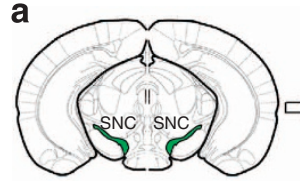

MPTP model

C
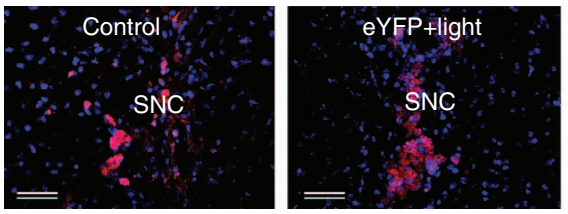

e
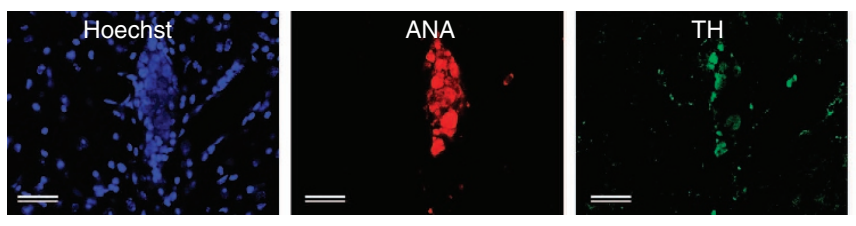

g
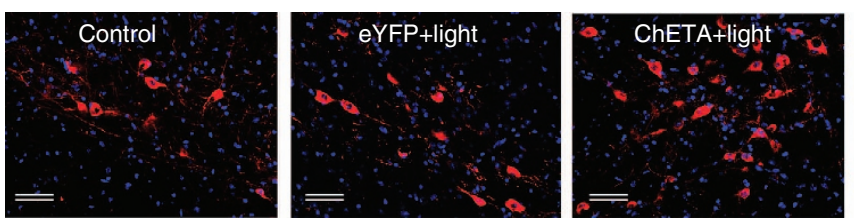

i

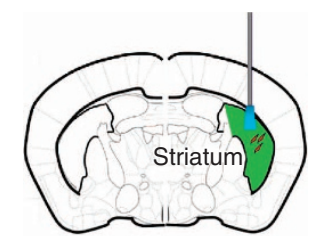

6-OHDA model

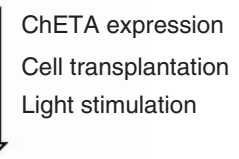

Rotation test j

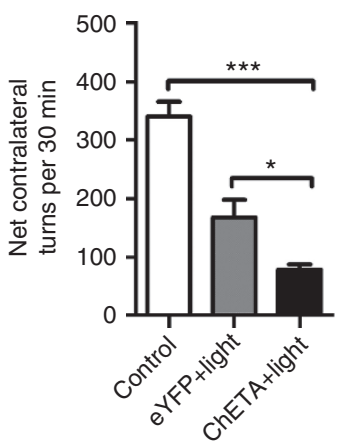

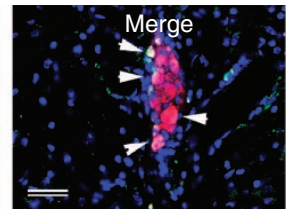

b

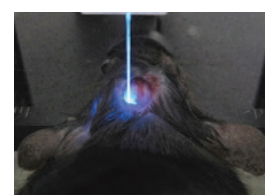

Light stimulation
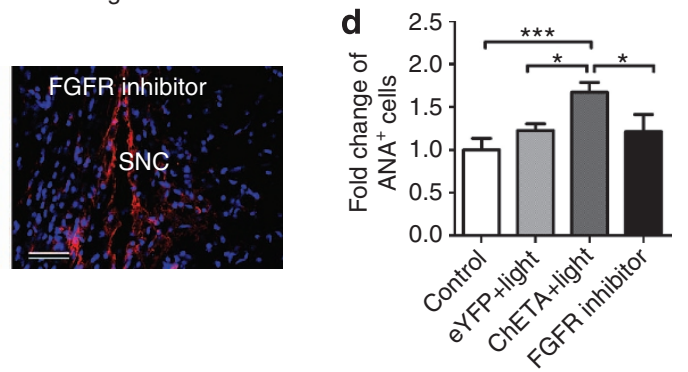

f

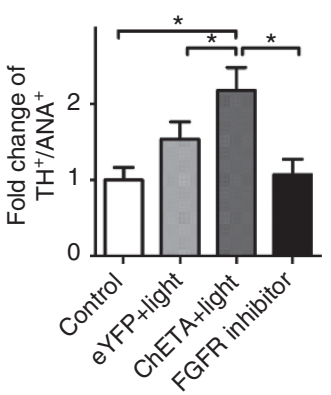

h
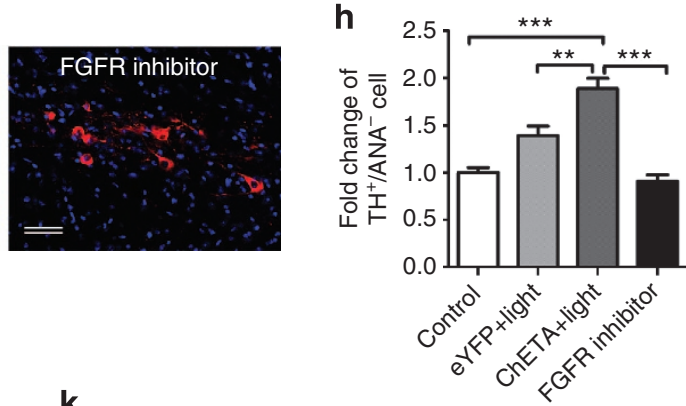

$\mathbf{k}$

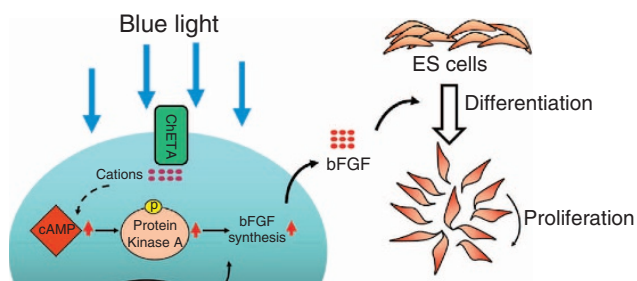

Neural progenitors

DDFF Differentiation

Astrocytes

xर

Dopamine neurons

Figure 6 | Light stimulation enhanced the dopaminergic differentiation of transplanted cells to promote brain repair. (a) Schematic flowchart of the experiment of stem cell transplantation and light stimulation. (b) The $S N$ region ( $A P=-3.28, M L=-1.5, D V=4.3$ ) was illuminated by blue light after the mice were anaesthetized. (c) ANA-positive cells were observed in the Control, eYFP + light, ChETA + light and FGFR Inhibitor groups. The signals in the ChETA + light group increased compared with the Control, eYFP + light and FGFR inhibitor groups. Bars, $50 \mu m$. (d) The fold change of ANA-positive cells in the Control, eYFP + light, ChETA + light and FGFR Inhibitor groups (mean \pm s.e.m., $n=8$ ). (e) Immunostaining of TH (green) and ANA (red) of the transplanted stem cells in the SN region. Arrow head, dual stained neurons. Bars, $20 \mu \mathrm{m}$. (f) The fold change of TH/ANA-positive cells in the Control, eYFP + light, ChETA + light and FGFR Inhibitor groups (mean \pm s.e.m., $n=8$ ). (g) Immunostaining of endogenous TH-positive cells in the Control, eYFP + light, ChETA + light and FGFR Inhibitor groups. Bars, $50 \mu \mathrm{m}$. (h) The fold change of endogenous TH-positive cells in the Control, eYFP + light, ChETA + light and FGFR Inhibitor groups (mean \pm s.e.m., $n=8$ ). (i) Schematic flowchart of the experiment of stem cell transplantation and light stimulation in 6-OHDA murine model. (j) Apomorphine-induced net contralateral turns in the Control, eYFP + light and ChETA + light groups. (mean \pm s.e.m., $n=5$ ). (k) Schematic drawing for the molecular mechanism of the light induced bFGF release to enhance the neural/dopaminergic differentiation of human embryonic stem cells. All analyses based on Student's $t$-test. ${ }^{\star} P<0.05$; ${ }^{\star \star} P<0.01$; ${ }^{\star \star \star} P<0.001$. The experiments were repeated three times with similar results. 
of the astrocytes and stem cell transplantation. Animals received Lentivirus-mediated in vivo transfection of ChETA, followed by stem cell transplantation and light stimulation (Fig. 6i). We found that the ChETA + light group showed significantly reduced net contralateral turns in an apomorphine-induced rotational behaviour test compared with Control and eYFP + light groups (Fig. 6j, Supplementary Movie 1), suggesting that ChETA expression and light stimulation were beneficial to the functional improvement in the 6-OHDA-induced PD model. Taken together, our data showed that light-activated endogenous astrocytes could enhance the DA differentiation of transplanted stem cells, increase the number of endogenous $\mathrm{TH}$-positive cells in SN and ameliorate the behaviour deficits in the PD model.

\section{Discussion}

In the current study we investigated the effects of optogenetically activated astrocytes on the DA differentiation of human ESCs and the underlying mechanisms, then we further explored the effects of specific activation of the endogenous astrocytes on the differentiation of transplanted stem cells and functional brain repair. Our study demonstrated, for the first time, that the optogenetically activated astrocytes in the SN upregulated the synthesis and release of bFGF, thereby enhancing the DA differentiation of human ESCs and improving brain repair in a mouse model of PD.

Astrocytes secrete a variety of neurotrophic factors to protect DA neurons and influence the neuronal differentiation of the stem cells. Electrical stimulation of cultured Schwann cells could induce a calcium-dependent release of nerve growth factor, which enhanced the survival of the neurons ${ }^{37}$. Treatment of astrocytes with apomorphine, a potent D1/D2 dopamine receptor agonist, enhanced the biosynthesis of multiple trophic growth factors including brain-derived neurotrophic factor and $\mathrm{bFGF}^{35}$, while selective phosphatidylinositol-linked D1-like receptor agonist upregulated the expression level of bFGF in striatal astrocytes in a dopamine D1/D2 receptor-independent manner ${ }^{21}$. To answer the question of whether astrocytes in the SN could be activated to synthesize and release bFGF, we applied optogenetics to regulate the activity of midbrain astrocytes, and found that light stimulation could significantly upregulate the synthesis and release of bFGF. Our co-transplantation assay showed that light stimulation could specifically upregulate the bFGF levels from transplanted astrocytes, demonstrated by the significantly increased bFGF levels in the ChETA + light group and decreased bFGF levels in the ChETA + light + siRNA group (Fig. 4). Using such a cell-type-specific stimulation approach, we further showed that light illumination of endogenous astrocytes could selectively increase the bFGF level in ChETA-transfected endogenous astrocytes (Fig. 5). Taken together, these findings demonstrated that optogenetic activation could specifically upregulate the bFGF level in astrocytes both in vitro and in vivo (Fig. 6k).

Previous findings have suggested that treatment of astrocytes with D1 receptor agonists stimulated PKA phosphorylation and activated the cAMP/PKA pathway ${ }^{35}$. Optogenetic manipulation of renal tubules could generate cAMP pulses rapidly and reversibly in a cell-type-specific manner ${ }^{38}$. In our study, elevated levels of PKA and CAMP were observed in the lightactivated astrocytes, consistent with our findings of upregulated expression of bFGF after the light illumination of astrocytes. Our evidence indicated that light-induced membrane depolarization could activate the cAMP/PKA pathway and increase the cAMP level in both cultured and endogenous astrocytes, thus providing an efficient strategy to directly regulate cAMP level and influence the intracellular signalling pathways in the astrocytes.
The FGF signalling pathway plays diverse roles in embryonic development, cell growth and carcinogenesis ${ }^{39}$. FGF-dependent signalling via phospho-Erk activation was also a major mediator of transitions in the lineage specification of the $\mathrm{ESCs}^{40}$, and blocking FGF signalling inhibited the neural induction of human ESCs $^{12}$. Specific and stage-dependent FGF-induced Erk1/2 signalling is also required for the neural specification of the $\mathrm{ESCs}^{41}$. Recently, an important study has also shown that increasing FGF signalling in the aged stem cell niche under homeostatic conditions could result in the loss of quiescence of stem cells and diminished regenerative capacity ${ }^{42}$. In our study, we first showed that bFGF derived from the light-activated astrocytes was not only required for the early neural differentiation of the human ESCs, but also needed to enhance the DA differentiation of neural progenitors at the late stage. The co-transplantation assay showed that bFGF derived specifically from transplanted astrocytes significantly enhanced the regenerative effects of stem cells. Last we used optogenetics to specifically regulate the endogenous astrocytes in the SN to promote in-situ bFGF release. We found that the elevated bFGF is necessary for enhancing the DA differentiation of the stem cells. This was supported by the findings of the increased expression of DA markers in the ChETA + light group but decreased expression in the presence of FGFR inhibitor. We observed the elevated $\mathrm{TH}$ signals in the striatm after intranigral transplantation of differentiated DA neurons or neural progenitors with astrocytes, suggesting that differentiated DA neurons in SN contributed to the increased DA axons and increased dopamine release in the striatum. Using three important approaches we consistently showed that bFGF derived from astrocytes played important and necessary roles to influence the differentiation and proliferation of the stem cells.

Besides the enhancing effects on the neural differentiation of the stem cells, bFGF could also protect nigral DA neurons from neurotoxin-induced cell death ${ }^{16}$. bFGF increased the BrdU-labelled proliferative cells in the SN of MPTP-treated mice ${ }^{43}$. Endogenous bFGF deficiency in FGF- $2^{-1-}$ mice caused a significant decrease in the DA neurons after neurotoxin application $^{44}$. Grafts of bFGF-expanded cells contained significantly more TH-positive cells than FGF-8 expanded cells, and induced a better functional recovery in 6-hydroxydopaminelesioned rats ${ }^{45}$. In our study we observed that the elevated bFGF promoted the proliferation and enhanced the DA differentiation of stem cells. Importantly, we also observed an elevated number of endogenous DA neurons and improved motor functions in the $\mathrm{SN}$ after light stimulation of the endogenous astrocytes. Our data suggested that boosting endogenous astrocyte activity through optogenetics could be beneficial to the survival of the remaining nigral DA neurons, elevated dopamine level and the amelioration of the behaviour deficits in the model of PD. It would also bring astrocytes as new therapeutic targets and help to ameliorate the neurodegenerative and behavioural effects in the treatment of PD.

Besides neurotrophic factors such as bFGF, recent studies have also shown that light stimulation could lead to gliotransmitter release from opsin-gene-expressing astrocytes. Optogenetically activated local astrocytes showed increased release of $\mathrm{ATP}^{46,4}$ and glutamate ${ }^{48}$. Optogenetic activation of astrocytes could also activate the neurons to trigger respiratory responses ${ }^{49}$, modulate motor behaviour ${ }^{48}$, enhance synaptic transmission ${ }^{50}$ and ameliorate ischemic brain damage ${ }^{51}$. On the basis of these studies we further showed that optogenetic activation of astrocytes could enhance the release of bFGF to promote the DA differentiation and functional repair in PD.

In conclusion, our study first discovered that the specific activation of midbrain astrocytes increased the synthesis and release of bFGF, which directed the differentiation of the 
transplanted hESCs to DA neurons.Using the optogenetic approach to regulate astrocytes in a tissue-specific manner would also be helpful to dissect the elaborate interactions between astrocytes and neurons in PD, which will contribute to the further understanding the biological role of the astrocytes in neural degenerative disease. Furthermore, clarification of the mechanisms of crosstalk between astrocytes, an important hosting cellular component, transplanted hESCs and the endogenous DA neurons will be crucial for an in-depth understanding of the development, survival and functional integration of DA neurons, and may provide the experimental evidence for novel therapeutic approaches for brain diseases involving dysfunction of DA neurons.

\begin{abstract}
Methods
ChETA expression in astrocytes using lentiviral vectors. Lentiviral vectors carrying the ChETA-eYFP or eYFP gene (Fig. 1b) were constructed. In brief, the $C M V$ or GFAP promoter was cloned into the pLenti-CaMK IIa-ChETA-eYFP or pLenti-CaMK IIa-eYFP plasmid (provided by Dr Karl Deisseroth in Stanford University). Then 293FT cells were transfected with the lentiviral vector $\mathrm{pCMV} \triangle \mathrm{R} 8.74$ and $\mathrm{pMD} 2 \mathrm{G}$, and a high titre lentivirus was produced. Twenty-four hours after the transfection, 293FT cells were transferred to serum-free medium containing $5 \mathrm{mM}$ sodium butyrate; the supernatant was collected and concentrated by ultracentrifugation at $50,000 \mathrm{~g}$, then phosphate-buffered saline (PBS) was used to re-suspend the viral pellets. Lentivirus was used to express the ChETA fusion protein in the cultured astrocytes. Primary cultured astrocytes were transfected with the Lentivirus $\left(10^{9} \mathrm{TU} \mathrm{ml}^{-1}\right)$ in a serum-free medium for $48 \mathrm{~h}$. Expression of ChETA-eYFP in astrocytes was monitored by observation of green fluorescent protein (GFP) expression. To evaluate the transfection efficiency, the astrocytes were counterstained with a GFAP antibody (Abcam). The ChETA-positive cells and GFAP-counterstained cells were counted in 10 high-magnification fields, and the percentage of transfected astrocytes over the GFAP-counterstained astrocytes was calculated.
\end{abstract}

In vitro stimulation of ChETA-expressing astrocytes. The primary rat striatal astrocytes were prepared from the striata of neonatal Sprague-Dawley rats. The neonatal striata were trypsinized for $10 \mathrm{~min}$ and tissues were dissociated and plated into six-well plates ${ }^{21}$. The astrocytes were divided into three groups: control group without ChETA expression and light stimulation; eYFP + light group, which expressed eYFP and was stimulated by light; and ChETA + light group, which expressed ChETA and was stimulated with light. A homemade light emitting diode (LED) was used to stimulate the astrocytes for $1 \mathrm{~h}$ using blue light with a wavelength of $460-485 \mathrm{~nm}$, and the average output power of the LED was $1.65 \mathrm{~mW} \mathrm{~mm}^{-2}$. At $24 \mathrm{~h}$ after the light stimulation, the CM from all the groups was harvested and used for inducing the neural differentiation of ESCs or other analyses.

In vitro culture and DA differentiation of hESCs. The experiments were approved by the Ethics Committee of the Shenzhen Institutes of Advanced Technology, ChineseAcademy of Sciences. The hESCs line H9 was kindly donated by Shenzhen Beike Biotechnology Company, and the culture of hESCs was performed as described previously ${ }^{33}$. In brief, the cells were grown on mouse embryonic fibroblasts and fed every $24 \mathrm{~h}$ with Knockout-DMEM (Invitrogen, Carlsbad, CA, USA) supplemented with $20 \% \mathrm{KO}$-serum replacement (KOmedium, Invitrogen). The hESCs were passaged every 7 days when the cells reached $80 \%$ confluence. At each passage, the cells were treated with collagenase type IV (Invitrogen) for $10 \mathrm{~min}$, and then the cells were gently scraped from the dish and split 1:4 among the coated culture plates

For the neural differentiation of hESCs, the cells were firstly dissociated to form embryonic bodies and suspended in culture for 4 days. The embryonic bodies were next plated on the dish in a medium containing insulin, transferrin and selenit (ITS medium, R\&D Systems, Minneapolis, MN, USA) and cultured for 9 days. Then the cells were trypsinized, transferred to the polyornithine + laminin-coated plates and cultured in DMEM/F12 supplemented with N2 medium (R\&D Systems) for 6 days; at this stage, the CM from different groups of activated astrocytes was added into the culture medium to evaluate the effects of $\mathrm{CM}$ on the neural differentiation of the hESCs. The DA differentiation of stem cells was further induced by replacing $\mathrm{N} 2$ medium with brain-derived neurotrophic factor $\left(25 \mathrm{ng} \mathrm{ml}^{-1}\right)$ and $0.5 \%$ fetal bovine serum(Invitrogen), and culturing the cells for 10 days. The medium in each group was changed three times per week and phase-contrast images were taken using a microscope (IX71, Olympus, Center Valley, PA, USA).

The cells were divided into three groups: the Control group was cultured in neural differentiation medium only; the eYFP + light group was cultured in half neural differentiation medium and half CM from eYFP-transfected astrocytes; the ChETA + light group was cultured in half neural differentiation medium and half CM from ChETA-eYFP-transfected astrocytes. The eYFP + light group was included in the assay to exclude non-specific effects of virus expression and light stimulation. The efficiency of neural/DA differentiation was examined by immunostaining using specific antibodies against nestin, Tujland tyrosine hydroxylase (TH).

For the co-culture of ES cells and transfected astrocytes, the stem cells were grown on top of a monolayer of astrocytes expressing ChETA-eYFP or eYFP alone (Supplementary Fig. 2a). The cells were divided into the Control group, eYFP + light group and ChETA + light group. The co-cultured cells were stimulated with a homemade LED for $1 \mathrm{~h}$ every 3 days using blue light with a wavelength of $460-485 \mathrm{~nm}$. The efficiency of neural/DA differentiation was examined by immunostaining of Nestin/Tuj1 at 15 or 35 days of differentiation.

Gene expression analysis using RT-PCR. At a late stage of DA differentiation, total RNAs were extracted from cells in Control, eYFP + light and ChETA + light groups using Trizol reagent. RT-PCR was performed using PrimeScript One Step RT-PCR Kit (Takara, Shiga, Japan) and $1 \mu \mathrm{g}$ of purified RNA. For real-time PCR, analysis was performed in triplicate using LightCycler480(Roche, Switzerland) with Thunderbird SYBR qPCR mix (Toyobo) and primers listed in Supplementary Table 1. Gene expression analysis was performed using the relative cycle threshold method, normalized to GAPDH expression, and the fold changes were calculated relative to the control group.

bFGF knockdown. Two separate standard siRNAs (Invitrogen), S132467 (sense: 5'-ACACUUACCGGUCACGGAATT-3'; antisense: 5'-UUCCGUGACCGGUA AGUGUTG- ${ }^{\prime}$ ) and S222026 (sense: $5^{\prime}$-GCCUGGAGUCCAAUAACUATT- $3^{\prime}$; antisense: $5^{\prime}$-UAGUUAUUGGACUCCAGGCGT- $3^{\prime}$ ) that target the sequences of rat $b F G F$ genes (GeneBank accession: NM_019305.2) were used to suppress the bFGF gene expression. The ChETA-transfected cells were divided into three groups: the Control group was treated with negative control siRNA; the siRNAl group was treated with S132467; and the siRNA2 group was treated with S222026. In brief, the transfection reagents (Invitrogen), $82 \mu \mathrm{l}$ culture medium without serum and antibiotics and $15 \mu \mathrm{l}$ of $5 \mu \mathrm{M}$ siRNA (S132467, S222026 or negative control siRNA, Invitrogen) were mixed together and incubated for 5-10 $\mathrm{min}$ at room temperature to allow the formation of transfection complexes, and then the complexes were added drop-wise onto the ChETA-expressing cells, giving a final siRNA concentration of $5 \mathrm{nM}$. The cells were incubated with the transfection complexes for $48 \mathrm{~h}$ under normal growth conditions without changing the medium. Cells in the three groups were stimulated with blue light for 1 hour and the protein was extracted $12 \mathrm{~h}$ after the light stimulation for western blot analysis.

Western blotting. Astrocytes were lysed and the protein was extracted using lysis buffer (NE-PERNuclear and Cytoplasmic Extraction Reagents, Pierce/Thermo Fisher Scientific, Waltham, MA, USA). Total proteins were determined using a Bicinchoninic Acid Protein Assay Kit (Pierce/Thermo Fisher Scientific). The samples were loaded onto $12 \%$ SDS-polyacrylamide gel electrophoresis separating gel and run at a voltage of $120 \mathrm{~V}$ for $2 \mathrm{~h}$. The total $\beta$-actin and bFGF contents were quantitated using mouse anti- $\beta$-actin antibodies (1:15,000, Sigma-Aldrich, St Louis, MO, USA) and rabbit anti-bFGF antibodies (1:100, Santa Cruz, Dallas, TX, USA), followed by horseradish peroxidase-conjugated goat anti-rabbit IgG (1:4,000, KPL, MD, USA). Colour development was achieved by using the ECL Western Blotting Detection Kit (Pierce/Thermo Fisher Scientific). Supplementary Figure 9 shows images of the full uncropped blots.

Quantitative determination of bFGF, cAMP and PKA. The concentrations of bFGF in cell extracts or tissue homogenate were determined using FGF basic ELISA Kit (Invitrogen). The concentrations of cAMP were determined using Cyclic AMP Direct EIA Kit (Arbor Assays, Ann Arbor, MI, USA). The concentrations of PKA were determined using PKA activity Kit (Arbor Assays). All the experiments were carried out according to the manufacturer's instructions. The optical density was determined at $450 \mathrm{~nm}$ using the microplate reader (Synergy 4, Winooski, VT, USA). The concentrations of bFGF, cAMP and PKA were determined, normalized to the Control group and compared among the different groups.

Immunostaining. Cultured cells were fixed with $4 \%$ paraformaldehyde (PFA), then incubated with mouse antibodies to SSEA3 (1:500, Invitrogen), SSEA4 (1:500, Invitrogen), Tra81 (1:500, Invitrogen), Tra60 (1:500, Invitrogen), Nestin (1:400, Sigma), Tuj1 (1:500, Sigma), TH (1:400, Millipore) or DAT (1:100, Santa Cruz) to characterize the molecular markers. After washing three times, the slides were incubated with FITC- or PE-conjugated secondary antibody (1:500, Invitrogen). Finally the sections were counterstained with 4',6-diamidino-2-phenylindole (DAPI) or Hoechst 33342 to stain the nuclei and then mounted.The number of Nestin-positive, Tuj1-positive, TH-positive and DAT-positive cells in each group was separately counted in 10 high-magnification fields; the percentage of positive cells was calculated and compared among different groups.

For the brain samples, the brains were fixed in $4 \%$ PFA at $4{ }^{\circ} \mathrm{C}$ overnight and cryosectioned at a thickness of $20 \mu \mathrm{m}$. The sections were then rehydrated and blocked by goat serum. The sections were incubated with primary antibodies to bFGF (1:200, Santa Cruz), nestin (1:400, Abcam, Cambridge, MA, USA), TH 
(1:500, Millipore, Billerica, MA, USA) or anti-nuclear antibody (ANA, 1:400, Millipore). The sections were then washed and labelled with the fluorescent secondary antibody (1:500, Invitrogen). The sections were counterstained with Hoechst 33342 and then mounted for image acquisition. The images were taken under the microscope (ECLIPSE 50i, Nikon, Melville, NY, USA). The number of TH-positiveor ANA-positive cells in the SN or striatum region was counted in 12 sections (three adjacent levels) from each mouse, and each group contained three mice. The mean cell number per section was normalized to the control group and compared among different groups.

EdU incorporation analysis for cell proliferation. The 5-ethynyl-2'-deoxyuridine (EdU) incorporation analysis was performed using a Click-iT Alexa Fluor 488 EdUImaging Kit (Invitrogen, Carlsbad, CA, USA). In brief, after 24-h incubation with $10 \mathrm{Mm}$ EdU, the cells were fixed with $4 \%$ PFA for $15 \mathrm{~min}$ and $0.5 \%$ Triton $\mathrm{X}-100$ was used to permeabilize the cells. The cells were incubated with the ClickiT reaction cocktail (Click-iT reaction buffer, CuSO4, Alexa Fluor 488 Azide, and reaction buffer additive) for $30 \mathrm{~min}$ while being protected from light. The cells were then incubated with Hoechst 33342 to stain the nuclei, and images were acquired. Eight images from each group were selected; the EdU-positive cells were counted using Image-Pro Plus software (Media Cybernetics, Rockville, MD, USA). The experiments were repeated three times for accuracy and the differences among the groups were compared.

\section{Establishment of the MPTP/6-OHDA model and cell transplantation. All} animal experimental protocols were approved by the Research Committee of the Shenzhen Institutes of Advanced Technology, Chinese Academy of Sciences. One hundred and ninety male C57 mice (6-8 weeks old) were used in the study. To establish the MPTP model of PD, C57 mice were given four intraperitoneal injections of 1-methyl-4-phenyl-1,2,3,6-tetrahydropyridine (MPTP, Sigma) at 2-h intervals, with a total dose per mouse of $100 \mathrm{mg} \mathrm{kg}^{-1}$. 6-hydroxydopamine (6-OHDA)-induced murine PD model was established as described previously ${ }^{52}$. One week after establishing the MPTP model, the mice were anaesthetized with an intraperitoneal injection of phenobarbitol sodium $\left(100 \mathrm{mg} \mathrm{kg}^{-1}\right)$. The head skin was cleaned with a $70 \%$ isopropanol solution and an incision was made along the midline. For the transplantation of differentiated DA neurons, the mice were divided into three groups: the Normal, MPTP Control and Treatment groups: the DA neurons $\left(2 \times 10^{5}\right)$ were injected into the $\mathrm{SN}$ region $(\mathrm{AP}=-3.28, \mathrm{ML}=-1.5$, $\mathrm{DV}=4.3$, ALLEN Mouse Brain Atlas) of Treatment groups. For the cell cotransplantation experiment, the mice were divided into three groups: the Control, eYFP + light and ChETA + light groups: the treated ChETA-transfected or eYFPtransfected astrocytes $\left(1 \times 10^{5}\right)$ and human ESCs-derived neural progenitors $\left(1 \times 10^{5}\right)$ were mixed in $5 \mu$ l DMEM medium and injected to the SN region $(\mathrm{AP}=-3.28, \mathrm{ML}=-1.5, \mathrm{DV}=4.3)$ or striatum region $(\mathrm{AP}=0.5, \mathrm{ML}=-2.0$, $\mathrm{DV}=4.3$ ) of ChETA + light group or eYFP + light group. For the stem cell implantation after the activation of endogenous astrocytes, the mice were divided into three groups: the Control, eYFP + light and ChETA + light groups: hESC-derived neural progenitors $\left(2 \times 10^{5}\right)$ were injected into the $\mathrm{SN}$ region $(\mathrm{AP}=-3.28, \mathrm{ML}=-1.5, \mathrm{DV}=4.3)$ of eYFP + light group and ChETA + light group. Experimenters were blinded to the treatment groups, sample sizes were based on previous experiments. Four mice were excluded from the analysis because of problems with the injection.

\section{In vivo transfection of endogenous astrocytes and light stimulation. One} week after establishing the MPTP mouse model, $3 \mu$ lentivirus solution $\left(10^{9} \mathrm{TU} \mathrm{ml}^{-1}\right)$ containing ChETA or eYFP vectors was injected into the $\mathrm{SN}$ region $(\mathrm{AP}=-3.28, \mathrm{ML}=-1.5, \mathrm{DV}=4.3)$ in the $\mathrm{ChETA}+$ light and eYFP + light groups, respectively; no virus was injected into the Control group. Three mice in the ChETA + light group were used to quantitate the percentage of ChETA-positive cells that were also GFAP-positive: the ChETA-positive or GFAP-positive cells were counted and summed up from 10 tissue sections separately. The number of GFAP-positive cells was expressed as a percentage of the total ChETA-positive cells.

One week after virus injection or cell transplantation, we used a blue-lightemitting optical fibre with a diameter of $200 \mu \mathrm{m}$ to illuminate the SN or striatal region with $100 \mathrm{~ms}$ pulses at intervals of $5 \mathrm{~min}$ for a total of $30 \mathrm{~min}$ in the eYFP + light and ChETA + light groups. The distance between the optical fibre tip and the cell implantation position was $\sim 100 \mu \mathrm{m}$ ( $\mathrm{SN}$ region: $\mathrm{AP}=-3.28$, $\mathrm{ML}=-1.5, \mathrm{DV}=4.2$; or striatum region: $\mathrm{AP}=0.5, \mathrm{ML}=-2.0, \mathrm{DV}=4.2$, and the average output power was $\sim 10 \mathrm{~mW} \mathrm{~mm}^{-2}$. The light stimulation was conducted once a week and lasted for 3 weeks in eYFP + light and ChETA + light groups. To inhibit the FGFR, $20 \mu \mathrm{M}$ FGFR inhibitor SU5402 (Tocris Bioscience, UK) was injected into the SN region before each light stimulation. One week after the last light stimulation, the mice were perfused with $4 \%$ PFA and the brain tissue was removed for bFGF quantification and immunostaining analysis.

Microdialysis and determination of dopamine levels. The mice were anaesthetized with sodium pentobarbital $\left(100 \mathrm{mg} \mathrm{kg}^{-1}\right)$ and placed in a stereotaxic frame. A microdialysis probe (MER-10 mm guide, $2 \mathrm{~mm}$ membrane, Bioanalytical Systems) was implanted into the striatum region $(\mathrm{AP}=0.5, \mathrm{ML}=-2.0$,
$\mathrm{DV}=4.3)$. The probes were perfused with artificial cerebrospinal fluid $(\mathrm{NaCl}$ $124 \mathrm{mM}, \mathrm{KCl} 3 \mathrm{mM}, \mathrm{CaCl}_{2} 2.4 \mathrm{mM}, \mathrm{MgSO}_{4} 1.3 \mathrm{mM}$, glucose $10 \mathrm{mM}$ and HEPES $10 \mathrm{mM}, \mathrm{pH}=7.3$ ) for $90 \mathrm{~min}$ before beginning the experiment, and samples were collected for $30 \mathrm{~min}$ in the experiment. The concentrations of dopamine in the dialysate were determined using a dopamine ELISA Kit (Abnova, Walnut, CA USA), and the experiments were carried out according to the manufacturer's instructions. In brief, dopamine in the dialysate is extracted using a cis-diol-specific gel, acylated and derivatized enzymatically. The optical density was determined at $450 \mathrm{~nm}$ using the microplate reader (Synergy 4, Biotek). The concentrations of dopamine were determined, normalized to that of the control group and compared among the different groups.

Electrophysiology. Whole-cell recordings of astrocytes and DA neurons were performed using standard whole-cell patch-clamp techniques. For astrocytes, a Multiclamp 700B amplifier (Molecular Devices, Sunnyvale, CA, USA) was used to acquire cell recordings. The currents were low-pass filtered at $1 \mathrm{KHz}$ and digitized at $10 \mathrm{KHz}$ using a Digidata 1440A (Molecular Devices). A standard $\mathrm{KCl}$ pipette solution containing the following salts was used: $140 \mathrm{mM} \mathrm{KCl}, 4.3 \mathrm{mM} \mathrm{MgCl}_{2}$, $11 \mathrm{mM}$ EGTA, $4.4 \mathrm{mM} \mathrm{Na} \mathrm{AT}_{2} \mathrm{ATP}$, and $10 \mathrm{mM}$ HEPES sodium salt; the $\mathrm{pH}$ was adjusted to 7.2 using Tris base. Osmolarity was adjusted to $302 \mathrm{mOsm} \mathrm{kg}^{-1}$. Normal extracellular $\mathrm{NaCl}$ bath solution contained the following salts: $150 \mathrm{mM}$ $\mathrm{NaCl}, 5 \mathrm{mM} \mathrm{KCl}, 3.31 \mathrm{mM} \mathrm{CaCl}, 10 \mathrm{mM}$ glucose and $10 \mathrm{mM}$ HEPES acid. The $\mathrm{pH}$ was adjusted to 7.4 and the osmolarity was $306-312 \mathrm{mOsm} \mathrm{kg}^{-1}$. We used a DG-4 high-speed optical switch with a $300 \mathrm{~W}$ xenon lamp (Sutter Instruments, Novato, CA, USA) to deliver the light pulses to the ChETA-activated astrocytes. The output power was $3-5 \mathrm{Mw}$, and we used $10-\mathrm{Hz}$ light stimulation $(1-500 \mathrm{~ms})$. The interval for the paired stimuli was $25-500 \mathrm{~ms}$. We acquired the data using pClamp10software (Molecular Devices).

Recordings from DA neurons were identified by the action potential and the presence of a large inward current (Ih, $>200 \mathrm{pA}$ ), evoked by a 1.5-s hyperpolarizing step from -50 to $-150 \mathrm{mV}$. Whole-cell voltage-clamp recordings were made at a holding potential of $-50 \mathrm{mV}$. The currents were filtered at $1 \mathrm{kHz}$, digitized at $2 \mathrm{kHz}$ and collected on a personal computer using pClampsoftware. The micropipettes were made from borosilicate glass capillaries, with a resistance in the range of 3-6 M $\Omega$. The pipette solutions used for whole-cell recordings contained $130 \mathrm{mMK}$-gluconate, $2 \mathrm{mM} \mathrm{MgCl}, 10 \mathrm{mM}$ HEPES, $1 \mathrm{mM}$ EGTA, $3.5 \mathrm{mMMg}$-ATP, $1 \mathrm{mM}$ Na-GTP and $10 \mathrm{mM}$ Na-phosphocreatine (pH $\left.7.3,280 \mathrm{mOsm} \mathrm{kg}^{-1}\right)$. The artificial cerebrospinal fluid contained $126 \mathrm{mMNaCl}$, $2.5 \mathrm{mMKCl}, 1.2 \mathrm{mM} \mathrm{NaH}_{2} \mathrm{PO}_{4}, 1.2 \mathrm{mMMgCl}_{2}, 2.4 \mathrm{mM} \mathrm{CaCl}_{2}, 11 \mathrm{mM}$ glucose and $21.4 \mathrm{mM} \mathrm{NaHCO}_{3}$, saturated with $95 \% \mathrm{O}_{2}-5 \% \mathrm{CO}_{2}\left(\mathrm{pH} 7.4,300 \mathrm{mOsm} \mathrm{kg}^{-1}\right.$ ).

Animal behaviour studies. For the grip strength test, forelimb grip strength was evaluated using a grip strength meter (Bioseb, France). The grip strength meter contains a digital force gauge attached to a grip strength platform having $3 \mathrm{~mm}$ size mesh. Mice in different groups were allowed to hold the platform with their forelimbs and pulled backwards with the tail in a horizontal plane. The maximum force applied to the platform immediately before the release of the paw grip was recorded in the meter. For the Pole Test, mice in different groups were placed vertically on a $30 \mathrm{~cm}$ vertical, $1 \mathrm{~cm}$ diameter pole. On the day before testing (day 1) the animals were habituated to the pole, and then the animals were recorded via digital video on the test day (day 2). The amounts of time were recorded for the mouse to turn towards the ground (time to orient down) and to reach the ground (time to descend). Rotational behaviour $\left(0.5 \mathrm{mg} \mathrm{kg}^{-1}\right.$ apomorphine, Sigma) was assessed at 4 weeks after the last light stimulation in a rotational system (Panlab, Spain), as previously described ${ }^{53}$. The results from the three different groups were expressed as contralateral net turns per $30 \mathrm{~min}$, and the difference was compared among the three groups.The Open-Field test consisted of an 8-min session in the open-field chamber $(50 \times 50 \mathrm{~cm})$, which was made of plastic and was divided into a central field (centre, $25 \times 25 \mathrm{~cm}^{2}$ ) and a periphery field. Each individual mouse from the different groups was placed in the periphery field at the start of the test. Behaviours were recorded on video during the trial and the ANY-maze video tracking system (Stoelting, USA) was used for analysis.

Statistical analysis. The experiment data were expressed as the mean \pm s.e.m. of the number of tests stated. Statistical comparisons were made using either Student's $t$-test or analysis of variance (ANOVA) followed by Bonferroni's multiple comparison's post-hoc test, as indicated in the figure legends. All of the statistical tests were performed using the Statview (version 10.0, SPSS, Chicago, IL) program package or Prism 5.0 software. A $P$ value $<0.05$ was taken as statistical significance.

\section{References}

1. Kim, J. H. et al. Dopamine neurons derived from embryonic stem cells function in an animal model of Parkinson's disease. Nature 418, 50-56 (2002).

2. Ganat, Y. M. et al. Identification of embryonic stem cell-derived midbrain dopaminergic neurons for engraftment. J. Clin. Invest. 122, 2928-2939 (2012). 
3. Kriks, S. et al. Dopamine neurons derived from human ES cells efficiently engraft in animal models of Parkinson's disease. Nature 480, 547-551 (2011).

4. Bayly, R. D., Brown, C. Y. \& Agarwala, S. A novel role for FOXA2 and SHH in organizing midbrain signaling centers. Dev. Biol. 369, 32-42 (2012).

5. Andersson, E. et al. Identification of intrinsic determinants of midbrain dopamine neurons. Cell 124, 393-405 (2006).

6. L'Episcopo, F. et al. Wnt/beta-catenin signaling is required to rescue midbrain dopaminergic progenitors and promote neurorepair in ageing mouse model of Parkinson's disease. Stem Cells 32, 2147-2163 (2014).

7. Joksimovic, M. \& Awatramani, R. Wnt/beta-catenin signaling in midbrain dopaminergic neuron specification and neurogenesis. J. Mol. Cell Biol. 6, 27-33 (2014).

8. Andersson, E. R. et al. Wnt5a cooperates with canonical Wnts to generate midbrain dopaminergic neurons in vivo and in stem cells. Proc. Natl Acad. Sci. USA 110, E602-E610 (2013).

9. Lahti, L., Peltopuro, P., Piepponen, T. P. \& Partanen, J. Cell-autonomous FGF signaling regulates anteroposterior patterning and neuronal differentiation in the mesodiencephalic dopaminergic progenitor domain. Development 139, 894-905 (2012).

10. Baron, O., Ratzka, A. \& Grothe, C. Fibroblast growth factor 2 regulates adequate nigrostriatal pathway formation in mice. J. Comp. Neurol. 520, 3949-3961 (2012).

11. Ye, W., Shimamura, K., Rubenstein, J. L., Hynes, M. A. \& Rosenthal, A. FGF and Shh signals control dopaminergic and serotonergic cell fate in the anterior neural plate. Cell 93, 755-766 (1998).

12. LaVaute, T. M. et al. Regulation of neural specification from human embryonic stem cells by BMP and FGF. Stem Cells 27, 1741-1749 (2009).

13. Cho, M. S., Hwang, D. Y. \& Kim, D. W. Efficient derivation of functional dopaminergic neurons from human embryonic stem cells on a large scale. Nat. Protoc. 3, 1888-1894 (2008).

14. Daadi, M. M. \& Weiss, S. Generation of tyrosine hydroxylase-producing neurons from precursors of the embryonic and adult forebrain. J. Neurosci. 19, 4484-4497 (1999).

15. Saarimaki-Vire, J. et al. Fibroblast growth factor receptors cooperate to regulate neural progenitor properties in the developing midbrain and hindbrain. J. Neurosci. 27, 8581-8592 (2007).

16. Grothe, C. \& Timmer, M. The physiological and pharmacological role of basic fibroblast growth factor in the dopaminergic nigrostriatal system. Brain Res. Rev. 54, 80-91 (2007).

17. Tooyama, I. et al. Loss of basic fibroblast growth factor in substantia nigra neurons in Parkinson's disease. Neurology 43, 372-376 (1993).

18. Timmer, M. et al. Enhanced survival, reinnervation, and functional recovery of intrastriatal dopamine grafts co-transplanted with Schwann cells overexpressing high molecular weight FGF-2 isoforms. Exp. Neurol. 187, 118-136 (2004).

19. Takayama, H. et al. Basic fibroblast growth factor increases dopaminergic graft survival and function in a rat model of Parkinson's disease. Nat. Med. 1, 53-58 (1995).

20. Bean, A. J. et al. Expression of acidic and basic fibroblast growth factors in the substantia nigra of rat, monkey, and human. Proc. Natl Acad. Sci. USA 88, 10237-10241 (1991).

21. Zhang, X. et al. Activation of phosphatidylinositol-linked D1-like receptor modulates FGF-2 expression in astrocytes via IP3-dependent $\mathrm{Ca} 2+$ signaling. J. Neurosci. 29, 7766-7775 (2009).

22. Robel, S., Berninger, B. \& Gotz, M. The stem cell potential of glia: lessons from reactive gliosis. Nat. Rev. Neurosci. 12, 88-104 (2011).

23. Li, K. et al. Ventral mesencephalon astrocytes are more efficient than those of other regions in inducing dopaminergic neurons through higher expression level of TGF-beta3. J. Mol. Neurosci. 37, 288-300 (2009).

24. Song, H., Stevens, C. F. \& Gage, F. H. Astroglia induce neurogenesis from adult neural stem cells. Nature 417, 39-44 (2002).

25. Marchetti, B. et al. Uncovering novel actors in astrocyte-neuron crosstalk in Parkinson's disease: the Wnt/beta-catenin signaling cascade as the common final pathway for neuroprotection and self-repair. Eur. J. Neurosci. 37, 1550-1563 (2013).

26. L'Episcopo, F. et al. Reactive astrocytes and Wnt/beta-catenin signaling link nigrostriatal injury to repair in 1-methyl-4-phenyl-1,2,3,6-tetrahydropyridine model of Parkinson's disease. Neurobiol. Dis. 41, 508-527 (2011).

27. L'Episcopo, F. et al. A Wnt1 regulated Frizzled-1/beta-Catenin signaling pathway as a candidate regulatory circuit controlling mesencephalic dopaminergic neuron-astrocyte crosstalk: Therapeutical relevance for neuron survival and neuroprotection. Mol. Neurodegener. 6, 49 (2011).

28. Sandhu, J. K. et al. Astrocyte-secreted GDNF and glutathione antioxidant system protect neurons against 6OHDA cytotoxicity. Neurobiol. Dis. 33, 405-414 (2009)

29. Chen, P. C. et al. Nrf2-mediated neuroprotection in the MPTP mouse model of Parkinson's disease: Critical role for the astrocyte. Proc. Natl Acad. Sci. USA 106, 2933-2938 (2009).
30. Chen, P. S. et al. Valproate protects dopaminergic neurons in midbrain neuron/ glia cultures by stimulating the release of neurotrophic factors from astrocytes. Mol. Psychiatry 11, 1116-1125 (2006).

31. Shao, W. et al. Suppression of neuroinflammation by astrocytic dopamine D2 receptors via alphaB-crystallin. Nature 494, 90-94 (2013).

32. Saijo, K. et al. A Nurr1/CoREST pathway in microglia and astrocytes protects dopaminergic neurons from inflammation-induced death. Cell 137, 47-59 (2009).

33. Roy, N. S. et al. Functional engraftment of human ES cell-derived dopaminergic neurons enriched by coculture with telomerase-immortalized midbrain astrocytes. Nat. Med. 12, 1259-1268 (2006)

34. Ungless, M. A. \& Grace, A. A. Are you or aren't you? Challenges associated with physiologically identifying dopamine neurons. Trends. Neurosci. 35, 422-430 (2012).

35. Li, A. et al. Apomorphine-induced activation of dopamine receptors modulates FGF-2 expression in astrocytic cultures and promotes survival of dopaminergic neurons. FASEB J. 20, 1263-1265 (2006).

36. Nomura, T., Goritz, C., Catchpole, T., Henkemeyer, M. \& Frisen, J. EphB signaling controls lineage plasticity of adult neural stem cell niche cells. Cell Stem Cell 7, 730-743 (2010).

37. Huang, J., Ye, Z., Hu, X., Lu, L. \& Luo, Z. Electrical stimulation induces calcium-dependent release of NGF from cultured Schwann cells. Glia 58, 622-631 (2010).

38. Efetova, M. et al. Separate roles of PKA and EPAC in renal function unraveled by the optogenetic control of cAMP levels in vivo. J. Cell. Sci. 126, 778-788 (2012).

39. Boilly, B., Vercoutter-Edouart, A. S., Hondermarck, H., Nurcombe, V. \& Le Bourhis, X. FGF signals for cell proliferation and migration through different pathways. Cytokine Growth Factor Rev. 11, 295-302 (2000).

40. Villegas, S. N., Canham, M. \& Brickman, J. M. FGF signalling as a mediator of lineage transitions-evidence from embryonic stem cell differentiation. J. Cell. Biochem. 110, 10-20 (2010).

41. Stavridis, M. P., Lunn, J. S., Collins, B. J. \& Storey, K. G. A discrete period of FGF-induced Erk1/2 signalling is required for vertebrate neural specification. Development 134, 2889-2894 (2007).

42. Chakkalakal, J. V., Jones, K. M., Basson, M. A. \& Brack, A. S. The aged niche disrupts muscle stem cell quiescence. Nature 490, 355-360 (2012).

43. Peng, J., Xie, L., Jin, K., Greenberg, D. A. \& Andersen, J. K. Fibroblast growth factor 2 enhances striatal and nigral neurogenesis in the acute 1-methyl-4phenyl-1,2,3,6-tetrahydropyridine model of Parkinson's disease. Neuroscience 153, 664-670 (2008).

44. Timmer, M. et al. Fibroblast growth factor (FGF)-2 and FGF receptor 3 are required for the development of the substantia nigra, and FGF-2 plays a crucial role for the rescue of dopaminergic neurons after 6-hydroxydopamine lesion. J. Neurosci. 27, 459-471 (2007).

45. Jensen, P., Pedersen, E. G., Zimmer, J., Widmer, H. R. \& Meyer, M. Functional effect of FGF2- and FGF8-expanded ventral mesencephalic precursor cells in a rat model of Parkinson's disease. Brain Res. 1218, 13-20 (2008).

46. Tu, J. et al. Light-controlled astrocytes promote human mesenchymal stem cells toward neuronal differentiation and improve the neurological deficit in stroke rats. Glia 62, 106-121 (2014).

47. Chen, J. et al. Heterosynaptic long-term depression mediated by ATP released from astrocytes. Glia 61, 178-191 (2013).

48. Sasaki, T. et al. Application of an optogenetic byway for perturbing neuronal activity via glial photostimulation. Proc. Natl Acad. Sci. USA 109, 20720-20725 (2012).

49. Gourine, A. V. et al. Astrocytes control breathing through $\mathrm{pH}$-dependent release of ATP. Science 329, 571-575 (2010).

50. Perea, G., Yang, A., Boyden, E. S. \& Sur, M. Optogenetic astrocyte activation modulates response selectivity of visual cortex neurons in vivo. Nat. Commun. 5, 3262 (2014)

51. Beppu, K. et al. Optogenetic countering of glial acidosis suppresses glial glutamate release and ischemic brain damage. Neuron 81, 314-320 (2014).

52. Chen, X. et al. Disrupted and transgenic urate oxidase alter urate and dopaminergic neurodegeneration. Proc. Natl Acad. Sci. USA 110, 300-305 (2013).

53. Xiao, D. et al. Forebrain adenosine A2A receptors contribute to L-3,4dihydroxyphenylalanine-induced dyskinesia in hemiparkinsonian mice. J. Neurosci. 26, 13548-13555 (2006).

\section{Acknowledgements}

This project was partially supported by the National Basic Research Program of China (2012CB966902, 2010CB529605 and 2011CB504400); the National Natural Science Foundation of China (91132306 and 81000551); the Instrument Developing Project (2010019), Strategic Priority ResearchProgram (B) of the Chinese Academy of Sciences (XDB02050003); Guangdong Innovation Research Team Fund for Low-cost Healthcare Technologies; Shenzhen Peacock Plan (KQCX20130628112914294), Shenzhen Governmental Basic Research Grants (JC201006040897A, JC201005270296A, and 
JC201005270291A). We thank Dr Heather J. Ballard, University of Hong Kong, for valuable comments on this manuscript.

\section{Author contributions}

L.W. and F.Y. conceived and designed the experiments. Y.L., J.T., J.W., J.Z., B.W. and S.C. performed experiments. F.Y., Y.L. and J.T. analysed data. J.Z. and Y.M. helped to design the experiments and provided assistance.

\section{Additional information}

Supplementary Information accompanies this paper at http://www.nature.com/ naturecommunications

Competing financial interests: The authors declare no competing financial interest.
Reprints and permission information is available online at http://npg.nature.com/ reprintsandpermissions/

How to cite this article: Yang, F. et al. Activated astrocytes enhance the dopaminergic differentiation of stem cells and promote brain repair through bFGF. Nat. Commun. 5:5627 doi: 10.1038/ncomms6627 (2014).

(c) (i) This work is licensed under a Creative Commons Attribution 4.0 International License. The images or other third party material in this article are included in the article's Creative Commons license, unless indicated otherwise in the credit line; if the material is not included under the Creative Commons license, users will need to obtain permission from the license holder to reproduce the material. To view a copy of this license, visit http://creativecommons.org/licenses/by/4.0/ 